\title{
Fechnerian metrics in unidimensional and multidimensional stimulus spaces
}

\author{
EHTIBAR N. DZHAFAROV \\ Hanse-Wissenschaftskolleg, Delmenhorst, Germany \\ and \\ HANS COLONIUS \\ University of Oldenburg, Oldenburg, Germany
}

\begin{abstract}
A new theory is proposed for subjective (Fechnerian) distances among stimuli in a continuous stimulus space of arbitrary dimensionality. Each stimulus in such a space is associated with a psychometric function that determines probabilities with which it is discriminated from other stimuli, and a certain measure of its discriminability from its infinitesimally close neighboring stimuli is computed from the shape of this psychometric function in the vicinity of its minimum. This measure of discriminability can be integrated along any path connecting any two points in the stimulus space, yielding the psychometric length of this path. The Fechnerian distance between two stimuli is defined as the infimum of the psychometric lengths of all paths connecting the two stimuli. For a broad class of models defining the dichotomy of response bias versus discriminability, the Fechnerian distances are invariant under response bias changes. In the case in which physically multidimensional stimuli are discriminated along some unidimensional subjective attribute, a systematic construction of the Fechnerian metric leads to a resolution of the long-standing controversy related to the numbers of just-noticeable differences between isosensitivity curves. It is argued that for unidimensional stimulus continua, the proposed theory is close to the intended meaning of Fechner's original theory.
\end{abstract}

\section{Introduction}

This paper presents a comprehensive theory of how subjective distances among stimuli belonging to a continuous $n$-dimensional stimulus space (such as a space of parametrized colors, spatial locations, or geometric shapes) can be computed from the degree of discriminability of each stimulus from its infinitesimally close neighboring stimuli. We term such a metric Fechnerian, because it appears that, in the case of unidimensional stimulus continua $(n=1)$, this idea, rather than the cumulation of finite "subjectively equal just-noticeable differences," constitutes the core of Fechner's original theory (Fechner, 1851, $1860,1877,1887)$. To the extent our interpretation of Fechner's work is correct, this paper refines and expands (to stimulus spaces of arbitrary dimensionality) the oldest theoretical construct in scientific psychology.

1.1. Background. In his book of rejoinders to his numerous critics, Fechner (1877, Postscript) compares his theory to the biblical tower of Babel: as the latter was not built because its builders could not agree on how to build it, so the edifice of his theory will not be torn down because his critics will not agree on how to tear it down. In

The authors are grateful to Hilel Gauchman for important suggestions. The authors thank A. A. J. Marley, R. Schweickert, J. Townsend, and two anonymous reviewers for their help in the revision of the first draft of this paper. Correspondence should be addressed to E. N. Dzhafarov, who is now at the Department of Psychological Sciences, Purdue University, 1364 Psychological Sciences Bldg., West Lafayette, IN 47907-1364. (e-mail: ehtibar@psych.purdue.edu). spite of Stevens's derision (Fechner's "edifice," he writes, was never torn down, it was merely abandoned; Stevens, 1975, p. 8), the history of psychophysics, prominently including the period following Stevens's work, proves Fechner's prophesy remarkably accurate. It would suffice to look through numerous critical commentaries on the recent papers by Krueger (1989), Lockhead (1992), and Murray (1992) or through recent monographs by Baird (1997), Laming (1997), and Link (1992) to see that Fechner's ideas are still very much in the center of psychophysical controversies. The most prominent controversy is focused on Fechner's logarithmic "psychophysical law" versus its competitors, but this is one issue that concerns us very little in this paper. The focus of the paper is instead on the essential logic of the Fechnerian scaling, one of the criteria of the essential logic being its extendability to multidimensional stimulus spaces. The traditionally considered "psychophysical laws" have little relevance beyond unidimensional stimulus continua, and, even there, our approach is consistent with the possibility that a correct computation of Fechnerian distances for different continua and different experimental conditions leads to different "psychophysical laws."

The aspect of Fechner's legacy that is of primary importance for this paper is his proposal (or our interpretation thereof) to measure the subjective distance between stimuli $a$ and $b$ by "moving" a stimulus $x$ from $a$ to $b$ and "cumulating" along the way the degree of discriminability of $x$ from its neighboring stimuli (see Figure 1). (Fechner, as most psychophysicists after him, preferred to fix $a$ at 


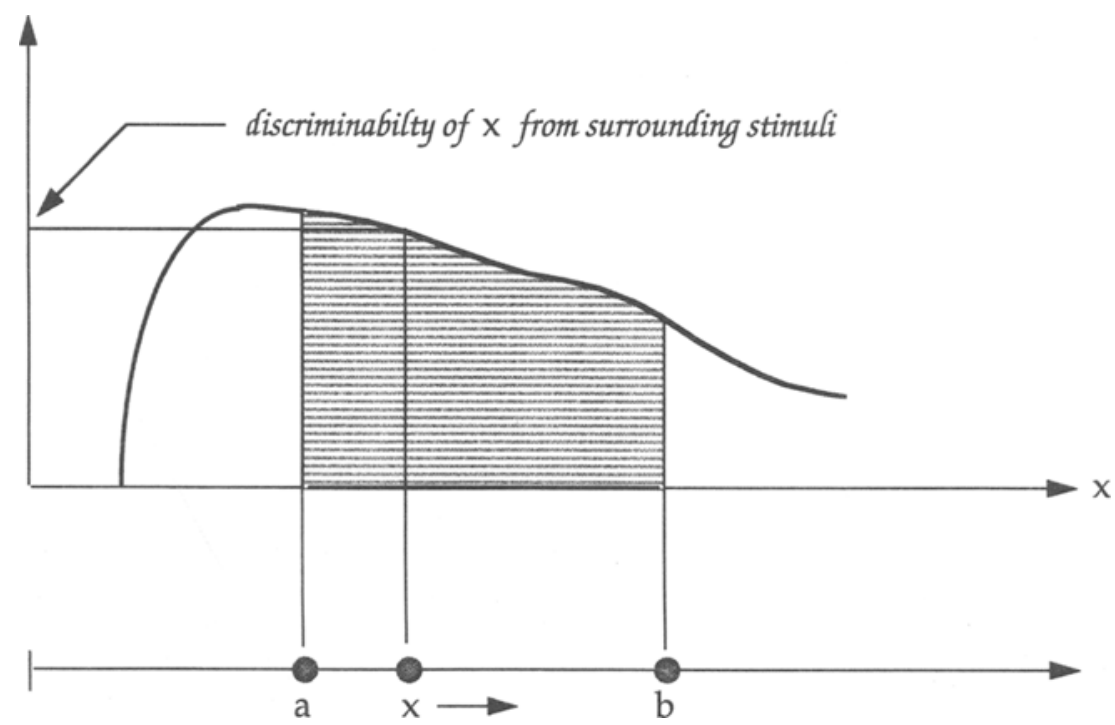

Figure 1. The logic of unidimensional Fechnerian scaling: subjective distance between $a$ and $b$ is the integral of a measure of the discriminability of $x$ from its neighboring stimuli.

the value of the absolute threshold [or any physical value below it, say, zero] and think of the subjective distance from this point to $b$ as the magnitude of the "sensation" caused by $b$. This approach, however, is only applicable to unidimensional continua with a leftmost zero, because of which it is not considered in this paper.) Fechner's belief that the subjective distances computed by means of the cumulation of a local discriminability measure are more basic than those measured by any other means is easily understandable: The ability to perceptually distinguish different stimuli is arguably the only one shared by all perceiving organisms, and it is prerequisite for all other cognitive abilities.

The specific choice of the local discriminability function and the way of its cumulation proposed by Fechner have been criticized throughout the history of psychophysics, by the contemporaries of Fechner's and ours alike. The traditional understanding (analyzed later in detail) is that Fechner equates the discriminability of a stimulus $x$ from its neighbors with the reciprocal of the finite justnoticeable difference (JND) with respect to $x$ and integrates this value from $a$ to $b$ to obtain the subjective distance between these stimuli. This procedure is believed to rest on Fechner's postulate that stimuli separated by equal numbers of JNDs are subjectively equidistant. One line of criticism (described later) points out that the logic of this procedure is internally inconsistent (Elsass, 1886; Luce \& Edwards, 1958). The prevailing view among modern psychophysicists (Falmagne, 1971, 1985; Luce \& Galanter, 1963) is that this procedure should be forsworn in favor of a related but different scaling problem: how to assign numerical values to unidimensional stimuli so that these values are equidistant for stimuli discriminated with equal probabilities (this is referred to as the "Fechner problem," although Guilford, 1954, p. 39, attributes the under- lying probability-distance principle to Fullerton and Cattell). Below, we propose an interpretation of Fechner's theory that embeds it in our general theory of Fechnerian scaling (in stimulus spaces of arbitrary dimensionality) and frees it from the internal inconsistencies found in it by Fechner's critics. According to this interpretation, Fechner's scaling theory is much more general than the modern solution of the Fechner problem: Fechnerian distances may very well violate the probability-distance principle.

Another line of criticism points out that Fechner's measurement procedure fails a crucial empirical test of internal consistency (Krueger, 1989; Laming, 1997; Stevens, 1936). Refer to Figure 2, in which two-dimensional stimuli (e.g., tones varying in intensity and frequency, or flashes varying in intensity and duration) that fall on the same curve are judged to have equal values of some subjective attribute, $\ell$ (loudness for tones, or brightness for flashes). Thus, the $\ell$ values of $\mathbf{a}_{1}$ and $\mathbf{b}_{1}$ equal the $\ell$ values of $\mathbf{a}_{2}$ and $\mathbf{b}_{2}$, respectively. According to the traditional interpretation of Fechnerian scaling, this implies that the number of the just-noticeable increments along the physical dimension $v$ that are required to get one from $\mathbf{a}_{1}$ to $\mathbf{a}_{2}$ must be the same as that for getting one from $\mathbf{b}_{1}$ to $\mathbf{b}_{2}$. Actual measurements (by Riesz, 1933, for tones; by Nachmias \& Steinman, 1965, for light flashes) do not accord with this prediction. A detailed analysis presented in this paper shows, however, that this prediction is based on assumptions that do not follow from the logic of Fechnerian scaling. In fact, the Fechnerian theory of unidimensional continua does not apply to the situation in question at all: One needs a more general theory of subjectively unidimensional discriminations among physically multidimensional stimuli. Once this theory is constructed, it becomes apparent that the Fechnerian distances in Figure 2 cannot be computed along the vertical lines and that, by construc- 


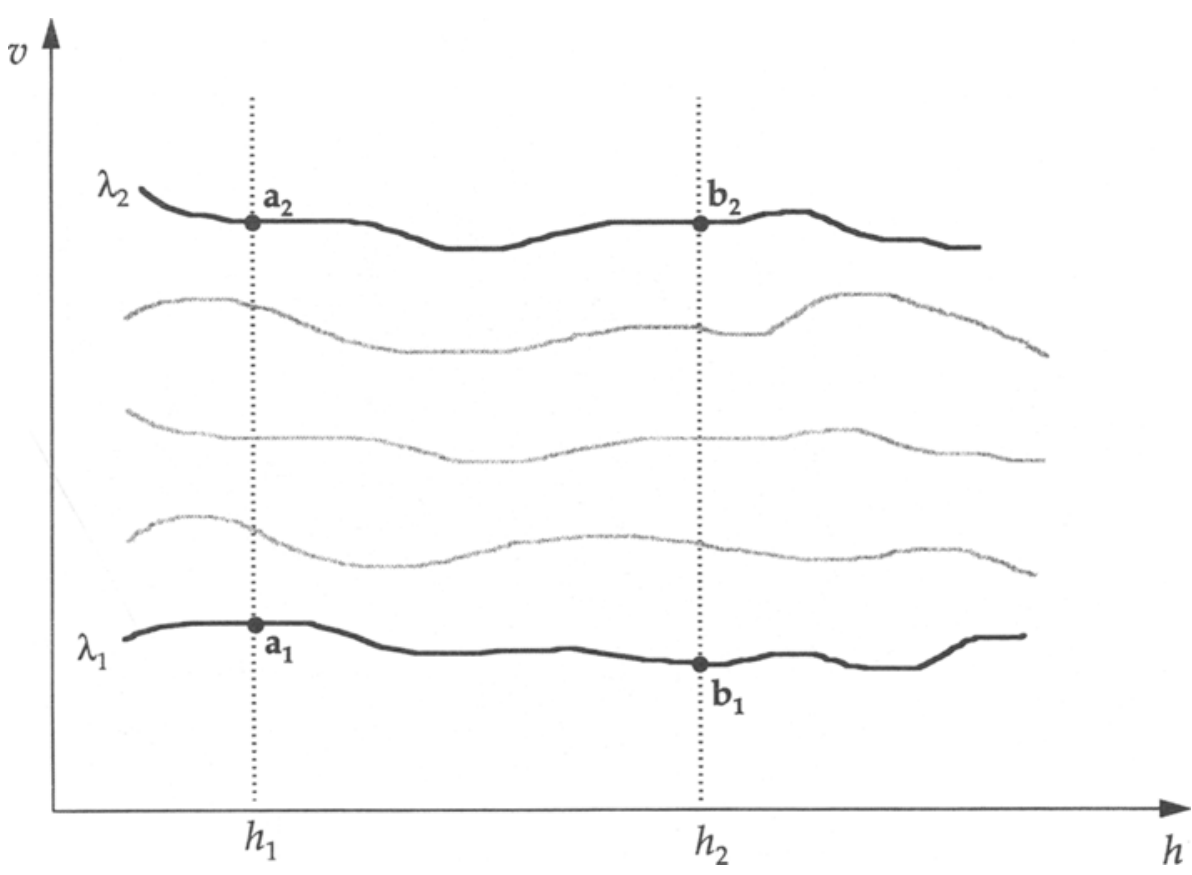

Figure 2. Isosensitivity curves in two dimensions $(h, v)$ discussed in Sections 1.1 and 6.2.

tion, the correctly computed Fechnerian distances from $\mathbf{a}_{1}$ to $\mathbf{a}_{2}$ and from $\mathbf{b}_{1}$ to $\mathbf{b}_{2}$ cannot be anything but equal. Concerning the proposed interpretation of Fechner's theory, we do not wish to imitate those who tend to find hidden depths in any sufficiently old text. By modern standards, Fechner was less than mathematically rigorous, and his writings are too voluminous and complex to dismiss the traditional interpretation of his theory with certainty. We cite evidence, however, that seems to indicate that our interpretation is closer to the intended meaning of what Fechner called his "psychophysical edifice." If so, our use of the term Fechnerian in describing the subjective metric developed in this paper is justified.

Our interpretation of Fechner's theory accords with Pfanzagl's (1962), Creelman's (1967), and Krantz's (1971) defense of Fechner's "differential formula" and, especially, the formulation by M. F. Norman mentioned in Krantz's paper as a personal communication. Among precursors of our generalization of Fechnerian scaling to multidimensional stimulus spaces, one can list Helmholtz's (1891) and Schrödinger's (1920) differential-geometric analysis of color metrics, as well as a recent work by Levin (in press).

1.2. Intuitive framework of the theory. To construct a general theory of Fechnerian scaling, applicable to stimulus spaces of arbitrary dimensionality, one has to answer two fundamental questions. The first one is how to compute the discriminability of a stimulus from its neighboring stimuli (which includes the question of what the precise meaning of "neighboring" is). The second question is how to cumulate this local discriminability measure along a transition path leading in the stimulus space from one stimulus to another (which includes the question of how one chooses these transition paths in multidimensional spaces). The intuitive points underlying our answers to these questions, and thereby our construction of the Fechnerian metric, are as follows.

(A) Refer to Figure 3. Any two $n$-dimensional stimuli $\mathbf{x}$ and $\mathbf{y}$ are associated with a discrimination probability

$$
\psi_{\mathbf{x}}(\mathbf{y})=\operatorname{Prob}\{\text { “ } \mathbf{y} \text { is different from } \mathbf{x} "\} \text {. }
$$

Nonidentical stimuli $\mathbf{x}$ and $\mathbf{y}$, however close, can be discriminated with a probability greater than the probability of discriminating $\mathbf{x}$ from itself:

$$
\begin{aligned}
\psi_{\mathbf{x}}(\mathbf{y}) & =\operatorname{Prob}\{“ \mathbf{y} \text { is different from } \mathbf{x} "\} \\
& >\text { Prob }\{\text { “x is different from } \mathbf{x} "\}=\psi_{\mathbf{x}}(\mathbf{x}) .
\end{aligned}
$$

(B) Refer to Figure 4. The infinitesimally small difference of discrimination probabilities,

$$
\psi_{\mathbf{x}}(\mathbf{x}+d \mathbf{x})-\psi_{\mathbf{x}}(\mathbf{x}),
$$

can be considered a "psychometric value" (an operationalist substitute for "subjective value") of the infinitesimally small difference between stimuli $\mathbf{x}$ and $\mathbf{x}+d \mathbf{x}$.

(C) Refer to Figure 5. Any sufficiently smooth path (curve) connecting two finitely distant stimuli $\mathbf{a}$ and $\mathbf{b}$ in the stimulus space can be viewed as a concatenation of infinitesimally small stimulus changes along this path. The corresponding psychometric values of these changes can be integrated and considered this path's "psychometric length." The length of the shortest path connecting $a$ and b can be considered the Fechnerian (subjective) distance between these two points. 


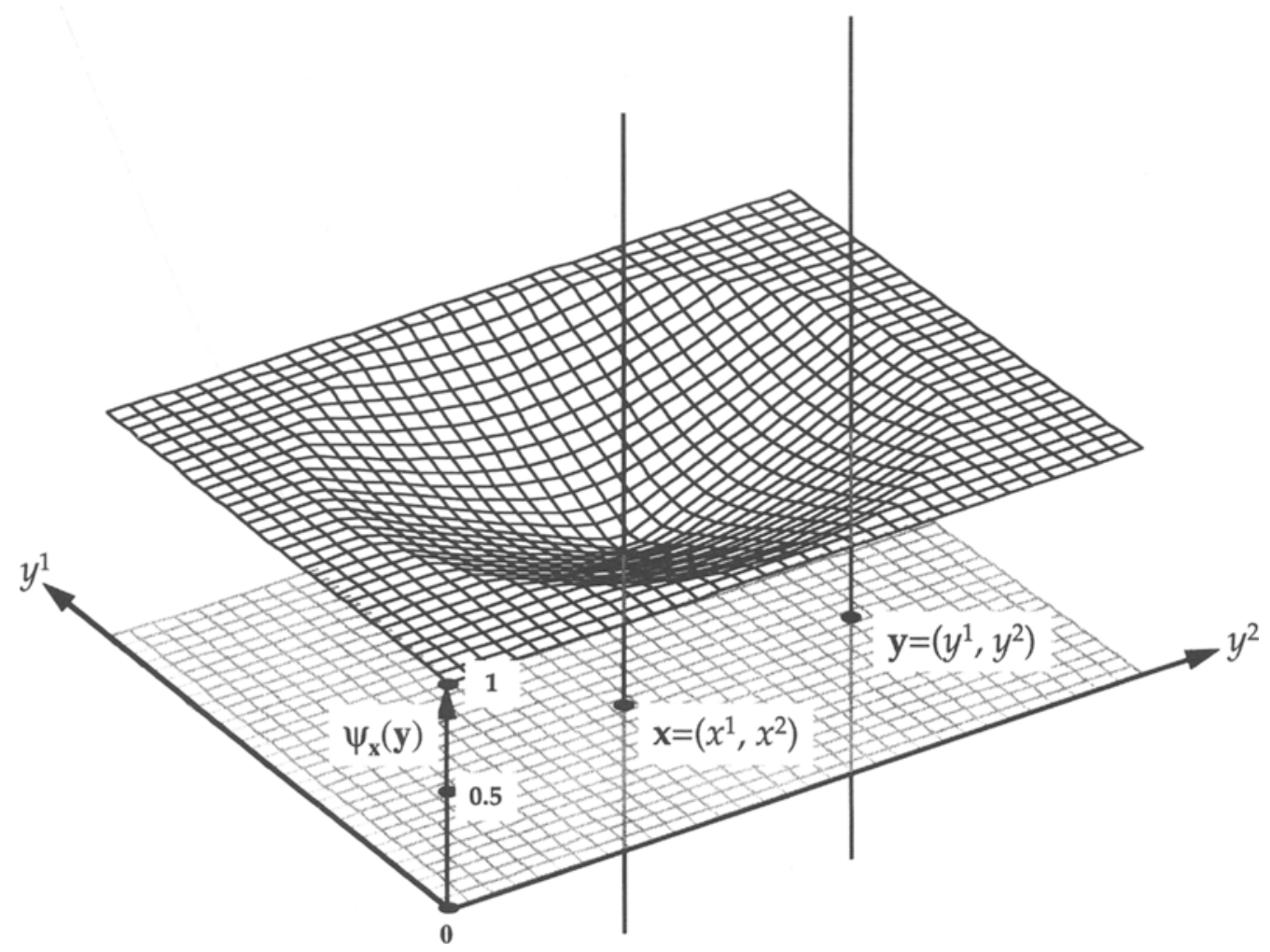

Figure 3. Possible appearance of a two-dimensional psychometric function $\psi_{x}(y)=$ Prob $\{$ " $y$ is different from $x "\} ; \psi_{x}(y)$ exceeds $\psi_{x}(x)$ for any $y \neq x$.

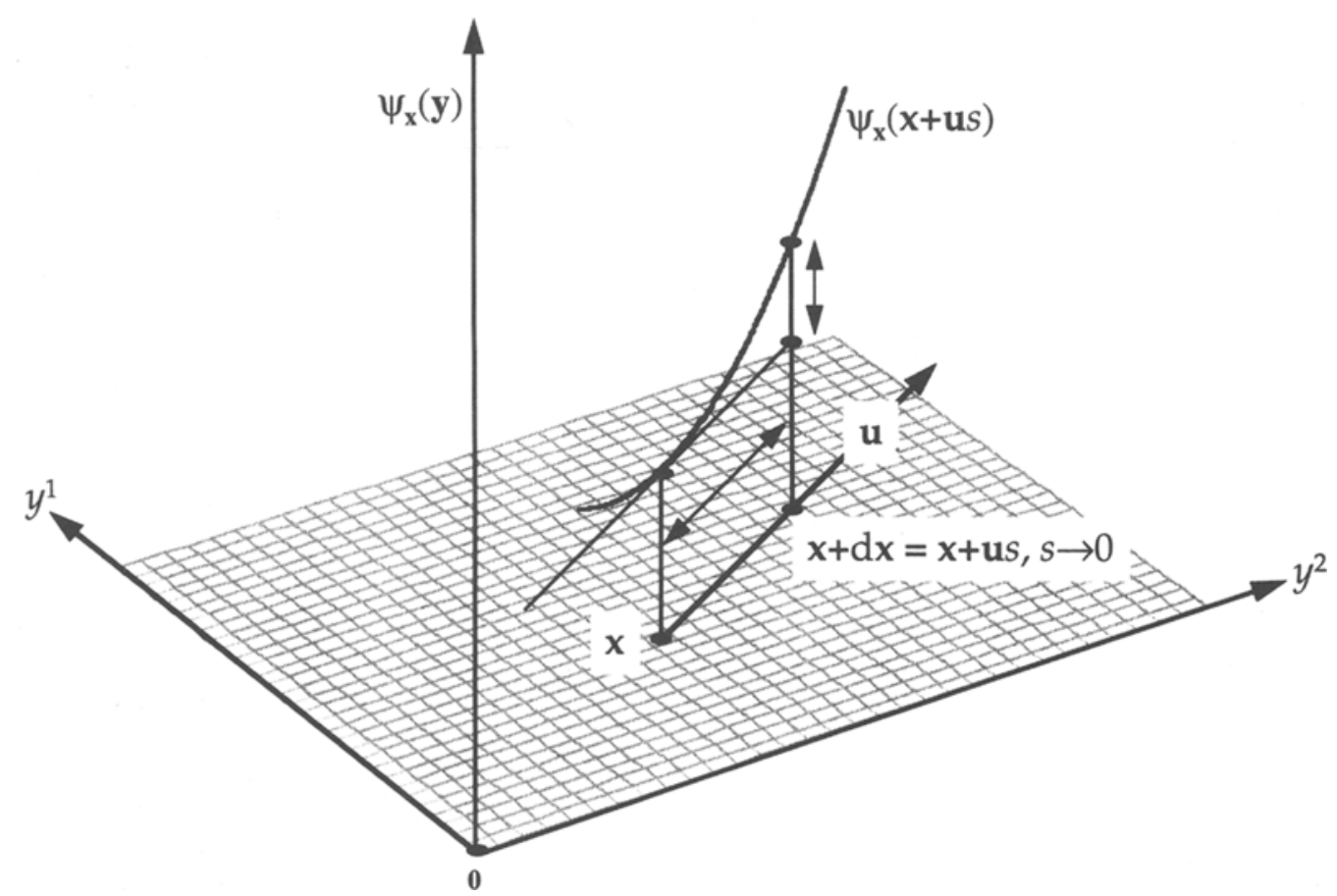

Figure 4. A small segment of the cross section of the psychometric function $\psi_{x}(y)$ of Figure 3 through its minimum (at $y=x$ ) along a direction $u$. As $s \rightarrow 0+$, the distance between $x$ and $x+u s$ (horizontal arrows) corresponds to the psychometric distance between $\psi_{x}(x)$ and $\psi_{x}(x+u s)$ (vertical arrows). 


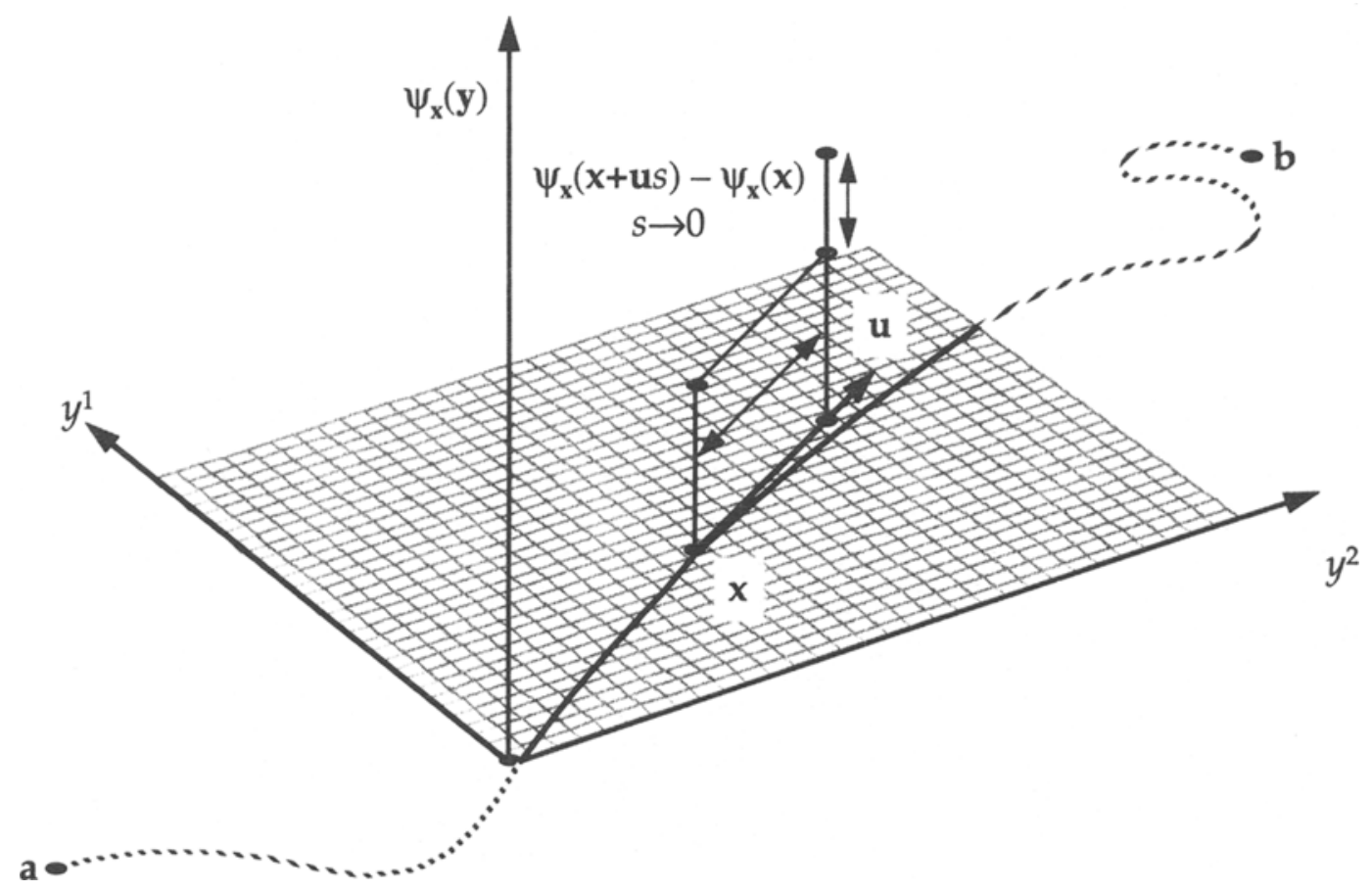

Figure 5. The subjective (or psychometric) length of a path connecting points a and $b$ is obtained by integrating along the path the psychometric differential $\psi_{\mathrm{x}}(\mathrm{x}+\mathrm{us})-\psi_{\mathrm{x}}(\mathrm{x}), s \rightarrow 0+$, corresponding to the infinitesimally small change from $x$ to $x+u s$ along the tangent direction $u$. (In a more general case, the psychometric differential should be replaced with some fixed transformation thereof.)

This is merely an intuitive framework: It is to be elaborated in many respects to form a comprehensive and mathematically rigorous theory. Some of these elaborations, briefly mentioned, are as follows.

(a) Psychometric functions $\psi_{\mathbf{x}}(\mathbf{y})$ need not be obtained by direct same-different judgments. There are alternative ways of obtaining functions interpretable as indicating degree of discriminability. From a strictly operationalist point of view, each procedure of computing $\psi_{\mathbf{x}}(\mathbf{y})$ may lead to its own Fechnerian metric, in one and the same stimulus space. Because of this, the qualifications used above, "subjective" and "psychometric," are relative, unless their equivalence across different judgment schemes is established empirically. It is also possible to relate the Fechnerian distances to unobservable "true" discrimination functions, about which it is assumed that the observable psychometric functions are their transforms affected by certain "response bias" parameters. One of the remarkable results established below is that, except for a scaling constant, the Fechnerian distances are invariant with respect to response bias changes for a wide class of conceivable response bias models.

(b) The requirement that $\psi_{\mathbf{x}}(\mathbf{y})$ achieve its minimum at $\mathbf{y}=\mathbf{x}$ is not critical. It is sufficient to assume that any psychometric function $\psi_{\mathbf{x}}(\mathbf{y})$ has a point of a global minimum, say, at $\mathbf{y}=\mathbf{x}^{*}$, and that the relationship between the reference stimuli $\mathbf{x}$ and the points of global minima $\mathbf{x}^{*}$ is one-to-one. The Fechnerian distance between two points, $\mathbf{a}$ and $\mathbf{b}$, is then defined as the Fechnerian distance between the two points of minima, $\mathbf{a}^{*}$ and $\mathbf{b}^{*}$, of the corresponding psychometric functions, $\psi_{\mathrm{a}}(\mathbf{y})$ and $\psi_{\mathrm{b}}(\mathbf{y})$. In the important case (mentioned in the previous section) when physically $n$-dimensional stimuli are discriminated along a unidimensional subjective attribute, the Fechnerian distances are computed among $(n-1)$-dimensional hypersurfaces (lines, if $n=2$ ) at which the psychometric functions reach their minima.

(c) The physical distance between $\mathbf{x}+d \mathbf{x}$ and $\mathbf{x}$ can be presented as the (positive) infinitesimal $d s$ in the representation

$$
\mathbf{x}+d \mathbf{x}=\mathbf{x}+\mathbf{u} d s, d s>0,
$$

which means that $\mathbf{x}+d \mathbf{x}$ lies at a distance $d s$ from $\mathbf{x}$ in a direction $\mathbf{u}$. The corresponding psychometric differential

$$
\psi_{\mathbf{x}}(\mathbf{x}+d \mathbf{x})-\psi_{\mathbf{x}}(\mathbf{x})=\psi_{\mathbf{x}}(\mathbf{x}+\mathbf{u} d s)-\psi_{\mathbf{x}}(\mathbf{x})
$$

serves as a measure of discriminability (of $\mathbf{x}+\mathbf{u} d s$ from $\mathbf{x}$ ) only if it is comeasurable with $d s$ (i.e., has the same order of infinitesimality, as explained in Section 2). In other cases, such a measure is provided by a monotonic transformation of this differential,

$$
\Phi\left[\psi_{\mathbf{x}}(\mathbf{x}+d \mathbf{x})-\psi_{\mathbf{x}}(\mathbf{x})\right]
$$

where $\Phi$ is assumed to be the same for all psychometric functions $\psi_{\mathbf{x}}(\mathbf{y})$ and all directions $\mathbf{u}$ of the infinitesimal change $d \mathbf{x}$. Except for a scaling constant, this transformation is determined asymptotically uniquely (i.e., uniquely in the vicinity of zero, the only region where it 
is needed). For simplicity and without much loss of generality, throughout most of the paper, the transformation $\Phi$ is assumed to be a power function.

(d) The computation of Fechnerian distances is not predicated on any assumptions about the global structure of the psychometric functions $\psi_{\mathbf{x}}(\mathbf{y})$. One does not need even such elementary assumptions as the symmetry,

$$
\psi_{\mathbf{x}}(\mathbf{y})=\psi_{\mathbf{y}}(\mathbf{x})
$$

or the continuity of $\psi_{\mathbf{x}}(\mathbf{y})$ at all points. Figure 6 provides an exaggerated illustration of this point: The three contours of the psychometric functions shown are all admissible. It is assumed, however, that any psychometric function has a point of a global minimum, and certain regularity conditions are imposed on the shape of the psychometric functions in the vicinity of their global minima.

(e) The psychometrically "shortest" path connecting two stimuli need not exist: The Fechnerian distance between $\mathbf{a}$ and $\mathbf{b}$ can be merely the greatest lower boundary for the length of all paths connecting these stimuli. The properties of the shape of the psychometric functions in the vicinity of their minima guarantee the existence of this greatest lower boundary.

(f) The computation of Fechnerian distances is a problem of variational analysis. It leads to the differentialgeometric structure called the Finslerian geometry, the more familiar Riemannian geometry being its special case. (This paper, however, does not presuppose a prior knowledge of variational calculus, differential geometry, or any other branch of mathematics beyond standard calculus of several variables.) It should be emphasized that the Finslerian geometry is not assumed here for mathematical reasons, such as simplicity or generality, and certainly not because the Riemannian metric appears to us too restrictive (see Section 7.3). In fact, the Finslerian geometry in our work is not assumed at all, rather it follows from the logic of computing the local discriminability from the psychometric functions.

1.3. Epistemological status of Fechnerian scaling. Fechnerian scaling is not a mathematical technique but an empirical theory based on assumptions that may or may not be true. It is not logically necessary that any two suf- ficiently close stimuli are associated with a fixed discrimination probability or that these probabilities form a psychometric function with a global minimum (it is conceivable, for example, that an observer would always judge any two stimuli different). Nor is it necessary that the form of the psychometric functions in the vicinity of their minima has the properties postulated below. At the same time, as these postulated properties relate to infinitesimally small areas of psychometric functions, to falsify or corroborate them, one would typically need a very large experiment that may only be feasible for lower dimensional stimulus spaces (perhaps only uni- or two-dimensional ones). Moreover, even with a very large experiment involving, say, two-dimensional stimuli, one cannot probably avoid the necessity of linking the differential properties of the psychometric functions being tested with some simplifying technical assumptions, thereby making the attribution of a potential falsification dubious. This situation, however, seems to be typical for psychological theories (see Dzhafarov, 1993).

In this paper, we avoid making such technical assumptions (e.g., parametric assumptions concerning the global form of the psychometric functions), because we do not believe that to introduce additional constraints with the sole purpose of facilitating the empirical falsifiability of a theory is good science: one needs compelling empirical or mathematical reasons for doing so, and we presently lack both. We see the main contribution of our work in the conceptual clarity that it brings to any area of research related to the intuitive notions of subjective metrics and subjective spaces, thereby providing a solid conceptual framework for interpreting empirical data and constructing empirically falsifiable models. The analysis of the Fechnerian distances between isosensitivity curves presented in Section 6.2 (mentioned earlier in the discussion related to Figure 2) is one example of how the conceptual clarity provided by our theory helps to resolve a longstanding theoretical controversy and save a good deal of experimental effort.

1.4. Structure of the paper. In Section 2 , we analyze the case of unidimensional stimulus continua and introduce some of the theoretical language to be utilized later

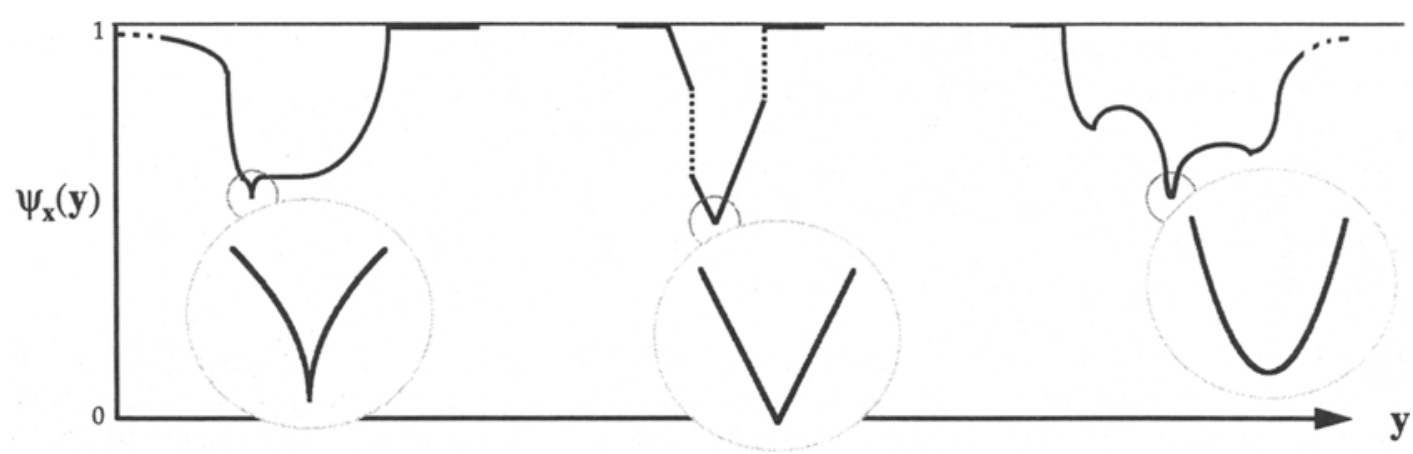

Figure 6. Fechnerian scaling imposes no restrictions on the global structure of psychometric functions, except in the infinitesimally small vicinity of their global minima. Shown are vertical cross sections of psychometric functions through their global minima. 
in the general theory. Section 3 is central for this paper, as it provides a comprehensive account of Fechnerian scaling for arbitrary $n$-dimensional continuous stimulus spaces. Some special cases of this theory are considered in Section 4. In Section 5, we discuss the dichotomy of "response bias" versus "true discriminability" and the problem of consistency between the models defining this dichotomy and Fechnerian scaling. In Section 6, we discuss the application of Fechnerian scaling to subjectively unidimensional discriminations of multidimensional stimuli. In Section 7, we outline possible generalizations of Fechnerian scaling, explain why the Fechnerian metric cannot be postulated to be Riemannian, and make concluding remarks. The Appendix is essentially a series of numbered endnotes containing technical comments and proofs; in the main text, they are referred to as A1-A7.

\section{Unidimensional Fechnerian Scaling}

2.1. Traditional interpretation of Fechner's approach. Fechner's $(1851,1860,1877,1887)$ original theory applies to stimuli that are measured on a single physical continuum and linearly ordered along a "corresponding" subjective dimension. The following is the gist of the traditional presentation of Fechner's theory in the psychophysical literature (see, e.g., Baird, 1997; Luce \& Edwards, 1958).

Fechner's theory (FT). Denoting stimulus magnitude by $x$, it is postulated that there is a real-valued function $\phi(x)$ ("sensation magnitude") such that the subjective distance $G(a, b)$ between two stimuli can be presented as

$$
G(a, b)=|\phi(b)-\phi(a)| .
$$

This is the subjective unidimensionality requirement, usually assumed tacitly. It is taken as an empirical fact that for any stimulus $x$, one can find a stimulus $x+\Delta x(\Delta x>0)$ that is just-noticeably greater than $x$. The dependence of $\Delta x$ on $x$,

$$
\Delta x=w(x),
$$

is referred to as the Weber function. The subjective distance $\Delta \phi$ between $x$ and $x+\Delta x$ is postulated to be constant across the stimulus continuum (Fechner's postulate):

$$
\Delta \phi=c .
$$

The two preceding equalities are combined into

$$
\frac{\Delta x}{w(x)}=\frac{\Delta \phi}{c},
$$

because both ratios equal unity. Then, a mathematical auxiliary principle is invoked, according to which the finite differences $\Delta x$ and $\Delta \phi$ can be replaced by differentials. This replacement yields Fechner's differential formula (Fundamentalformel)

$$
c \frac{d x}{w(x)}=d \phi(x) .
$$

The subjective distance $G(a, b)$ between two arbitrary stimuli is computed by integration of the above,

$$
G(a, b)=\phi(b)-\phi(a)=c \int_{a}^{b} \frac{d x}{w(x)}, a<b .
$$

This is Fechner's Unterschiedsformel (the more familiar Massformel being its special case, when $a$ is the absolute threshold).

The transition from the JNDs to differentials in FT is clearly unfounded and has been criticized by many. Elsass (1886), Fechner's contemporary, pointed out that the derivation is self-contradictory if the Weber law holds,

$$
w(x)=k x .
$$

In this case, the "derivation" above results in Fechner's logarithmic law,

$$
G(a, b)=\frac{c}{k} \log \frac{b}{a}, a<b,
$$

where $c$ is supposed to be the constant size of the subjective difference between $x$ and $x+k x$,

$$
G(x, x+k x)=\Delta \phi(x)=c .
$$

If, however, one computes $G(x, x+k x)$ directly from the logarithmic formula, one gets

$$
G(x, x+k x)=\frac{c}{k} \log (1+k) \neq c .
$$

Luce and Edwards (1958) provide a more general demonstration of the self-contradictory nature of (what is traditionally considered to be) Fechner's derivation, by pointing out that

$$
G(a, a+\Delta a)=c \int_{a}^{a+w(a)} \frac{d x}{w(x)}
$$

generally cannot be a constant, contrary to Fechner's postulate.

Fechner's critics also pointed out that the derivation above does not take into account that the Weber function $w(x)$ is not unique. A traditional recommendation (see Figure 7) is to estimate $w(x)$ by the distance

$$
w(x, p)=\gamma_{x}^{-1}(p)-\gamma_{x}^{-1}(1 / 2)=\gamma_{x}^{-1}(p)-x
$$

between $x$ (the median) and an arbitrary above-median quantile of the psychometric function

$$
\gamma_{x}(y)=\operatorname{Prob}\{\text { “ } y \text { is greater than } x "\} .
$$

(For simplicity and, as is shown below, with no loss of generality, we assume here that the median of a psychometric function equals the reference stimulus.) As there is no justification for preferring one quantile level $p$ to another, the Weber functions should be viewed as depending on both $x$ and $p$. It is easy to check, however, that if the subjective distance between two stimuli is computed as

$$
G(a, b)=c_{p} \int_{a}^{b} \frac{d x}{w(x, p)},
$$

then it would generally depend on $p$, and that, moreover, the values of $G(a, b)$ computed for two different values of 


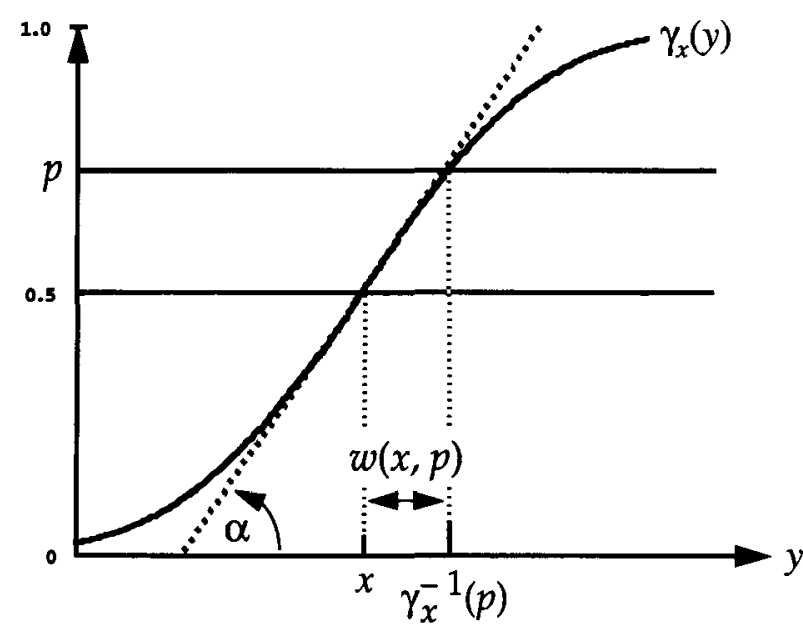

Figure 7. Possible appearance of a unidimensional psychometric function $\gamma_{x}(y)=\operatorname{Prob}\{$ “ $y$ is greater than $x$ " $\}$. A value of the Weber function $w(x, p)=\gamma_{x}^{-1}(p)-\gamma_{x}^{-1}(1 / 2)$ is shown by the arrows. The partial derivative $\bar{w}(x)$ of the Weber function with respect to $p$ at $p=1 / 2$ is reciprocal to the slope $\tan \alpha$ of the psychometric function at $p=1 / 2$.

$p$ would not generally be related to each other by any function.

For these reasons, the prevailing opinion is that the entire derivation associated with Fechner's differential formula should be dispensed with. Instead, it is suggested (Falmagne, 1971, 1985; Krantz, 1971; Levine, 1970; Luce \& Galanter, 1963; Pfanzagl, 1962) that the Fechnerian "sensation scale" $\phi(x)$ should be understood as a function satisfying the functional equation

$$
\phi[x+w(x, p)]-\phi(x)=g(p) .
$$

This equation formalizes the probability-distance principle mentioned in Section 1.1, and it can be viewed as a generalized version of Fechner's postulate (because of which Luce \& Galanter, 1963, refer to it as the "Fechner problem"): For any given $p$, the subjective difference corresponding to the "with-probability- $p$-noticeable difference" $w(x, p)$ is constant across the stimulus continuum. A comprehensive algebraic investigation of this functional equation can be found in Pfanzagl (1962) and, especially, Falmagne (1985).

This approach to Fechnerian scaling, however, has a surprising outcome, pointed out by Pfanzagl (1962): If a sufficiently smooth solution $\phi(x)$ for the functional equation representing the Fechner problem exists, then an expression closely resembling, if not identical to the rejected differential formula of Fechner's must hold. By differentiating the functional equation above with respect to $p$ at $p=1 / 2$, one gets

$$
\left.\phi^{\prime}(x) \frac{\partial w(x, p)}{\partial p}\right|_{p=1 / 2}=c .
$$

Denoting

$$
\bar{w}(x)=\left.\frac{\partial w(x, p)}{\partial p}\right|_{p=1 / 2}
$$

and assuming that this quantity is finite and nonzero (i.e., that the median of the psychometric function is a regular point of increase, as in Figure 7), we have

$$
d \phi(x)=c \frac{d x}{\bar{w}(x)} .
$$

We see that the modern reformulation of Fechnerian scaling implies Fechner's original differential formula, provided that $w(x)$ in FT is interpreted as $\bar{w}(x)$, the derivative of the Weber function $w(x, p)$ (with respect to $p$ at $p=1 / 2$ ) rather than the Weber function itself at a particular value of $p$. A similar point is made in Krantz (1971), although there it is formulated and arrived at in an unnecessarily complicated way.

Quite obviously, one can postulate the differential formula even if the functional equation representing the Fechner problem has no solution. The differential formula, therefore, represents a more general approach and can be introduced independently. A way of doing this can be derived from a personal communication by $M$. F. Norman reported in Krantz (1971). When modified to suit our purposes, Norman's formulation defines the Fechnerian sensation scale $\phi(x)$ as satisfying ${ }^{1}$

$$
\begin{aligned}
\phi[x+w(x, p)]-\phi(x)= & c(p-1 / 2) \\
& +o\{p-1 / 2\}, p \rightarrow 1 / 2(c>0) .
\end{aligned}
$$

Put somewhat loosely, the equation states that the subjective difference corresponding to the with probability$p$-noticeable difference $w(x, p)$ is constant across the stimulus continuum if $p$ is infinitesimally close to $1 / 2$. Norman's (modified) formulation is equivalent to

$$
\lim _{p \rightarrow 1 / 2} \frac{\phi[x+w(x, p)]-\phi(x)}{p-1 / 2}=c,
$$

and it can be viewed as a mathematically rigorous version of Fechner's postulate. Fechner's differential formula follows from this version of the postulate immediately (e.g., by L'Hopital's rule), under appropriate smoothness assumptions about the Weber function $w(x, p)$.

The subjective equality of finite JNDs, $w(x, p)$, then becomes merely a statement of practical approximation (or empirical estimation): For a fixed $p=p_{0}$, if $w\left(x, p_{0}\right)$ is sufficiently small, then

$$
\phi\left[x+w\left(x, p_{0}\right)\right]-\phi(x) \propto \frac{\phi\left[x+w\left(x, p_{0}\right)\right]-\phi(x)}{\left(p_{0}-1 / 2\right)} \approx c .
$$

Now we can replace the self-contradictory derivation (FT) with the following internally consistent theory of how to impose the Fechnerian metric on a unidimensional stimulus continuum.

Fechner's theory (FT*). Stimulus magnitude $x$ and sensation magnitude $\phi(x)$ are understood as above. It is taken as an empirical fact that for any stimulus $x$ (within a suit- 
ably chosen open interval) and any $0<p<1$, one can find a stimulus $y=x+w(x, p)$ that is judged to be greater than $x$ with probability $p ; w(x, p)$ is referred to as the Weber function. It is assumed that this function is continuously differentiable in both arguments (at least in the vicinity of $p=1 / 2$ ) and that

$$
w(x, 1 / 2)=0, \bar{w}(x)=\left.\frac{\partial w(x, p)}{\partial p}\right|_{p=1 / 2}>0 .
$$

It is postulated that

$$
\lim _{p \rightarrow 1 / 2} \frac{\phi[x+w(x, p)]-\phi(x)}{p-1 / 2}=c,
$$

where $c$ is the same for all stimulus values (the infinitesimal version of Fechner's postulate). It follows that (e.g., by L'Hopital's rule)

$$
d \phi(x)=c \frac{d x}{\bar{w}(x)},
$$

Fechner's differential formula. The subjective distance $G(a, b)$ between two arbitrary stimuli is obtained by integration of the above, yielding Fechner's Unterschiedsformel,

$$
G(a, b)=\phi(b)-\phi(a)=c \int_{a}^{b} \frac{d x}{\bar{w}(x)}, a<b .
$$

This integral is well defined under the smoothness conditions imposed on $w(x, p)$.

Clearly, Fechner did not present his theory in this form. Several indications, however, convince us that the intended meaning of Fechner's (mathematically imperfect) presentation was closer to $\mathrm{FT}^{*}$ than to the traditional account (FT). We believe that Fechner did not think of his differential formula as being derived (by means of an ad hoc "mathematical auxiliary principle") from a precise law, such as Weber's, relating finite differences in stimuli to finite differences in sensations. Rather he thought of the differential formula as a precise statement, and he thought of Weber's law as merely a finite-difference approximation. In his reply to the above-mentioned critique by Elsass (1886), Fechner explicitly rejected the validity of equating sensation differences with relative stimulus differences,

$$
\frac{\Delta x}{w(x)}=\frac{\Delta \phi}{c},
$$

unless both $\Delta x$ and $\Delta \phi$ are "very small" (Fechner, 1887, p. 167). We understand this as saying that even if the lefthand side of the equality above is a constant [e.g., even if Weber's law holds precisely, with $w(x)=k x$ ], the equality is not valid unless $\Delta x$ and $\Delta \phi$ are (or closely approximate) differentials. As argued convincingly by Scheerer (1987), when Fechner developed his differential formula in 1850 (precisely on October 22, now celebrated as the "Fechner Day"), he did not even mention JNDs and their subjective equality, because at that time he was not aware of Weber's law. As can best be seen from Fechner's own account given in Appendix II of the second volume of Zend-Avesta (1851), he considered the differential formula a precise hypothetical statement about the mathematical dependency between the mental and the physical:

If the strength of the physical activity underlying some mental activity at some given point in space and time is measured by its energy $\beta$ (energy understood in the sense of mechanics), and if its change, assuming an infinitely small part of time and space, is named $d \beta$, then the accompanying change in the intensity of the mental activity, to be estimated by feeling or in consciousness, is not proportional to the energy change $d \beta$, but to the relative change $d \beta / \beta$, and hence expressible by $k d \beta / \beta$, or by $d \beta / \beta$ itself, if we put $k=1$ once and for all. If the energy of some physical element at a definite point in space and time is given, then it will be possible by summation of a continuous series of absolute increments thereof, to end up with the energy of any other element (or of the same element) in any other point in space and time; and by corresponding summation of the accompanying relative increments, i.e., by means of the integral $\int d \beta / \beta$, to end up with the mental or psychical intensity of the same element, where the mental intensity of the initial element must be taken as known, because it serves for the determination of the constant of the integral. Thus, the required mental intensity $\gamma$ of the second element will be $\gamma=\log (\beta / b)$, where $b$ denotes the value of $\beta$ for which $\gamma=0$. (Fechner, 1851; translated by Scheerer, 1987, p. 203)

2.2. Unidimensional Fechnerian scaling. We now use the refined version of Fechner's original ideas $\left(\mathrm{FT}^{*}\right)$ to construct a systematic theory of Fechnerian scaling for unidimensional stimulus continua.

We begin by observing (see Figure 7) that the function

$$
\bar{w}(x)=\left.\frac{\partial w(x, p)}{\partial p}\right|_{p=1 / x}
$$

is reciprocal to the derivative of the psychometric function

$$
\gamma_{x}(y)=\operatorname{Prob}\{\text { “ } y \text { is greater than } x "\}
$$

computed at its median. That is,

$$
\frac{1}{\bar{w}(x)}=\left.\frac{d \gamma_{x}(y)}{d y}\right|_{y=x},
$$

where we continue to assume that the median equals the reference stimulus. Therefore, instead of presenting Fechner's differential formula as before,

$$
d \phi(x)=c \frac{d x}{\bar{w}(x)},
$$

one can rewrite it as

$$
d \phi(x)=c d \gamma_{x}(x)
$$

We see that the differential formula simply states that the infinitesimal subjective difference $d \phi(x)$ between $x$ and $x+d x$ is proportional to the corresponding infinitesimal change 


$$
d \gamma_{x}(x)=\gamma_{x}(x+d x)-\gamma_{x}(x)=\gamma_{x}(x+d x)-1 / 2
$$

of the psychometric function $\gamma_{x}(y)$. Since the sensation magnitude function, $\phi(x)$, is not independently defined, nothing prevents one from putting $c=1$ and simply identifying the subjective difference between $x$ and $x+d x$ with

$$
d \gamma_{x}(x)=\gamma_{x}(x+d x)-\gamma_{x}(x) .
$$

This makes both Fechner's postulate (in its infinitesimal version, $\mathrm{FT}^{*}$ ) and the differential formula redundant: They both reduce to a single proposal, to measure the subjective difference between $x$ and $x+d x$ by $d \gamma_{x}(x)$. The Fechnerian distance between two stimuli, $a$ and $b$, then should be measured as

$$
G(a, b)=\int_{a}^{b} d \gamma_{x}(x), a<b .
$$

We suggest that this surprisingly simple approach to $G(a, b)$ constitutes the essence of Fechnerian scaling for unidimensional continua.

The integral above can be presented as

$$
G(a, b)=\int_{a}^{b} F(x) d x, a<b,
$$

where

$$
F(x)=\left.\frac{d \gamma_{x}(y)}{d y}\right|_{y=x} .
$$

We observe that, in order for $G(a, b)$ to be well defined, it is sufficient for $F(x)$ to be finite, positive, and continuous. If these requirements are met, then $F(x)$ above is the simplest example of what we call the Fechner metric function, which we now proceed to define in a greater generality. ${ }^{2}$

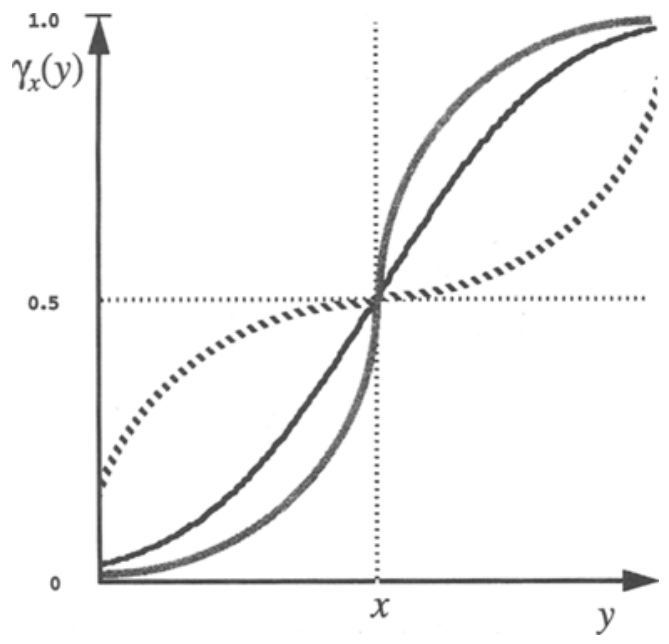

Figure 8. The slope of the tangent to the psychometric function $\gamma_{x}(y)$ at $p=1 / 2$ may be a positive number (solid dark line), zero (interrupted line), or infinity (gray line).
As is demonstrated in Figure 8, it may very well be the case that the derivative

$$
\left.\frac{d \gamma_{x}(y)}{d y}\right|_{y=x}
$$

equals zero or infinity. Put differently, it is possible that the infinitesimal

$$
\gamma_{x}(x+\Delta x)-\gamma_{x}(x), \Delta x \rightarrow 0
$$

has a higher or lower order of infinitesimality than the infinitesimal $\Delta x \rightarrow 0$. In such a case, it would make no sense to say that the subjective difference between $x$ and $x+d x$ can be measured by $d \gamma_{x}(x)$, since the two infinitesimals are not comeasurable. ${ }^{3}$ For any given $x$, however, there always exists an increasing transformation $\Phi\left[d \gamma_{x}(x)\right]$ that is comeasurable with $d x$ :

$$
\Phi\left[\gamma_{x}(x+\Delta x)-\gamma_{x}(x)\right]=O(\Delta x), \Delta x \rightarrow 0 .
$$

This trivially follows from the fact that the difference $\gamma_{x}(x+\Delta x)-\gamma_{x}(x)$ is an increasing function of $\Delta x$ in the vicinity of $\Delta x=0$.

Let us assume in the following that, in the vicinity of $\Delta x=0$, the psychometric function is odd-symmetrical,

$$
\lim _{\Delta x \rightarrow 0} \frac{\gamma_{x}(x+\Delta x)-\gamma_{x}(x)}{\gamma_{x}(x)-\gamma_{x}(x-\Delta x)}=1 .
$$

This assumption (that holds trivially if the function is differentiable at $\Delta x=0$ ) allows one to confine one's attention to positive differentials only,

$$
\Delta x \rightarrow 0+, \gamma_{x}(x+\Delta x)-\gamma_{x}(x) \rightarrow 0+.
$$

For simplicity, and with little loss of generality, one may assume that even if $\gamma_{x}(x+\Delta x)-\gamma_{x}(x)$ is not directly comeasurable with $\Delta x$, it is comeasurable with some power transformation $\Delta x^{\mu}$ of this differential $(\mu>0, \Delta x>0)$. This implies that the transformation $\Phi\left[\gamma_{x}(x+\Delta x)-\gamma_{x}(x)\right]$ can be taken to be the power function with the reciprocal exponent, and the comeasurability statement above can be presented as

$$
\sqrt[\mu]{\gamma_{x}(x+\Delta x)-\gamma_{x}(x)}=O(\Delta x), \Delta x \rightarrow 0+.
$$

To appreciate that this specialization of $\Phi$ is very unrestrictive, observe that it is satisfied whenever $\gamma_{x}(y)$ has a nonzero finite derivative of some order at $y=x$ : In this case, $\mu=m$, the lowest integer for which

$$
\left.\frac{d^{m} \gamma_{x}(y)}{d y^{m}}\right|_{y=x}>0
$$

(see Appendix A1). In a more general context, the possibility of nonpower transformations is discussed in Section 7.1, where it is shown that they do not lead to any nontrivial modifications in the theory.

A considerably more restrictive assumption we make now is that the exponent $\mu$ is one and the same for all 
psychometric functions-that is, for all values of $x .^{4}$ It follows that, for any stimulus $x$, the subjective distance between $x$ and $x+d x$ is comeasurable with (can be measured by)

$$
\sqrt[\mu]{\gamma_{x}(x+d x)-\gamma_{x}(x)}, d x>0 .
$$

We define the Fechner metric function as

$$
F(x)=\lim _{\Delta x \rightarrow 0+} \frac{\sqrt[4]{\gamma_{x}(x+\Delta x)-\gamma_{x}(x)}}{\Delta x}
$$

its value being, by construction, finite and positive. Assuming, in addition, that $F(x)$ is continuous, the existence of the Fechnerian distances

$$
G(a, b)=G(b, a)=\int_{a}^{b} F(x) d x, a<b
$$

is guaranteed. It is easy to check that $G(a, b)$ is a metric on the stimulus continuum: It is symmetrical, additive, and vanishes if and only if $a=b$.

So far, the construction of the Fechnerian metric has been predicated on the assumption that the median of a psychometric function equals the reference stimulus, $\gamma_{x}(x)=1 / 2$. In some experimental situations, this may not be true:

$$
\gamma_{x}^{-1}(1 / 2)=x^{*} \neq x
$$

The theory requires no nontrivial emendations to accommodate such cases, provided that the relationship between $x^{*}$ (the medians) and $x$ (the reference stimuli) is one-to-one (as one should expect it to be for all detectable stimuli). Indeed, then one can simply relabel the psychometric functions,

$$
\gamma_{x}(y)=\gamma^{*}{ }_{x^{*}}(y),
$$

and substitute $\gamma^{*}{ }_{x^{*}}\left(x^{*}+\Delta x^{*}\right)$ for $\gamma_{x}(x+\Delta x)$ in all formulations:

$$
F(x)=F^{*}\left(x^{*}\right)=\lim _{\Delta x^{*} \rightarrow 0+} \frac{\sqrt[\mu]{\gamma^{*}{ }_{x^{*}}\left(x^{*}+\Delta x^{*}\right)-\gamma^{*}{ }_{x^{*}}\left(x^{*}\right)}}{\Delta x^{*}}
$$

is finite, positive, and continuous in $x^{*}$, and

$$
G(a, b)=G^{*}\left(a^{*}, b^{*}\right)=\int_{a^{*}}^{b^{*}} F^{*}\left(x^{*}\right) d x^{*}, a^{*}<b^{*} .
$$

Unless mentioned specifically, we assume in this paper that such a relabeling has been made wherever necessary, and then the asterisks have been dropped for simplicity.

2.3. Psychometric functions in a canonical form. The Fechnerian metric as defined in the previous section is derived from the set of psychometric functions (indexed by $x$ )

$$
\gamma_{x}(y)=\operatorname{Prob}\{\text { " } y \text { is greater than } x "\} .
$$

These functions, however, cannot be utilized when dealing with subjectively multiattribute stimuli, which is normally the case when the stimuli are physically multidimensional and may happen even when the stimuli are physically unidimensional. It is important, therefore, to consider the Fechnerian metrics based on psychometric functions that do not presuppose subjective unidimensionality, such functions as

$$
\psi_{x}(y)=\operatorname{Prob}\{\text { “ } y \text { is different from } x "\}
$$

or

$$
\tilde{\psi}_{x}(y)=\operatorname{Prob}\{\text { “ distance between } x \text { and } y
$$

$$
\text { is greater than distance between } x \text { and } x "\} \text {. }
$$

Any such function can be interpreted as providing the probability with which $y$ can be discriminated from $x$, with no explicit specification of the subjective attributes involved. We assume that such a function has a single global minimum, which can always be made to be achieved at $y=x$ (by the relabeling argument analogous to that concluding Section 2.2). We refer to psychometric functions as being in a canonical form if they allow for such an interpretation and have the global minimum property just mentioned.

The term canonical form should not be taken to refer to any particular judgment scheme used in an experiment. The judgments defining $\psi_{x}(y)$ and $\tilde{\psi}_{x}(y)$ may lead to a canonical form directly, but this form can also be obtained, in a less direct way, from other judgment schemes. Notably, the psychometric function $\gamma_{x}(y)$ can be brought to a canonical form by interpreting the quantity

$$
1 / 2+\left|\gamma_{x}(y)-\gamma_{x}(x)\right|=\left|\gamma_{x}(y)-1 / 2\right|+1 / 2
$$

as indicating the probability with which $y$ can be discriminated from $x$ and by plotting this probability against $y$. This corresponds to the mirror-reflection procedure illustrated in Figure 9. For simplicity, a psychometric function in a canonical form is hereafter always presented as

$$
\psi_{x}(y)=\operatorname{Prob}\{\text { " } y \text { is different from } x "\},
$$

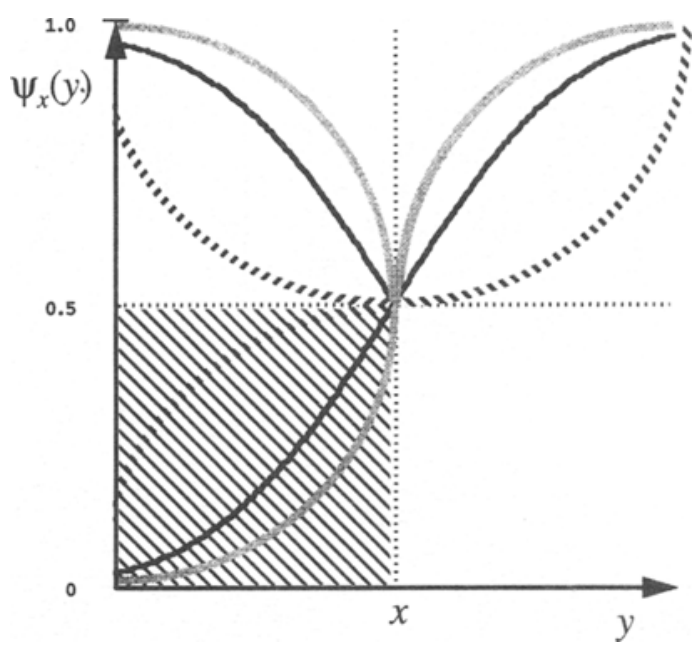

Figure 9. The canonical form $\psi_{x}(y)$ can be obtained from the psychometric function $\gamma_{x}(y)$ by mirror-reflecting its submedian part with respect to the median. 
whatever the factual judgment scheme and computational procedure by which this function is obtained.

Clearly, the mirror-reflection procedure is not the only logically possible way of transforming $\gamma_{x}(y)$ into a canonical form. We single out this procedure only because the Fechnerian metric derived from $\gamma_{x}(y)$ according to the previous section's computations then coincides with the Fechnerian metric derived from the canonical transformation $\psi_{x}(y)$ of $\gamma_{x}(y)$ according to the computations presented below. In general, unless empirically shown otherwise, it should be allowed that different judgment schemes and different procedures of bringing psychometric functions to a canonical form lead to different Fechnerian metrics.

We now proceed to deriving the Fechnerian metric from $\psi_{x}(y)$. We assume that the psychometric functions are (even-) symmetrical in the vicinity of their minima:

$$
\lim _{\Delta x \rightarrow 0} \frac{\psi_{x}(x+\Delta x)-\psi_{x}(x)}{\psi_{x}(x-\Delta x)-\psi_{x}(x)}=1 .
$$

This is the analogue of the odd-symmetry assumption of Section 2.2. We assume that there is a positive exponent $\mu$, one and the same for all stimuli $x$, such that

$$
\sqrt[\mu]{\psi_{x}(x+\Delta x)-\psi_{x}(x)}=O(\Delta x), \Delta x \rightarrow 0+.
$$

This is interpreted as justifying the proposal that the subjective distance between $x$ and $x+d x$ be measured by

$$
\sqrt[\mu]{\psi_{x}(x+d x)-\psi_{x}(x)}
$$

Figure 10 gives one an idea of the appearance of psychometric functions with different values of the exponent $\mu$.

The Fechner metric function, everywhere finite and positive, is introduced as

$$
F(x)=\lim _{\Delta x \rightarrow 0+} \frac{\sqrt[u]{\psi_{x}(x+\Delta x)-\psi_{x}(x)}}{\Delta x}
$$

and is assumed to be continuous. The Fechnerian distance

$$
G(a, b)=\int_{a}^{b} F(x) d x, a<b
$$

is then well defined and satisfies all requirements to a metric on a stimulus continuum.

Note that the minimum value $\psi_{x}(x)$ itself plays no role in the theory. It may or may not be the same for all $x$. For instance, it always equals $1 / 2$ when $\psi_{x}(x)$ is computed from $\gamma_{x}(x)$ by the mirror-reflection procedure illustrated in Figure 9 , but $\psi_{x}(x)$ may vary with $x$ if $\psi_{x}(y)$ is obtained by direct same-different judgments.

One of the most important properties of the Fechnerian distance is that it does not depend on the physical scale of measurement. Put more precisely, any two physical measurements, $x$ and $\bar{x}$, yield one and the same Fechnerian metric, provided that they are related by a positive diffeomorphism - that is, provided that the functions

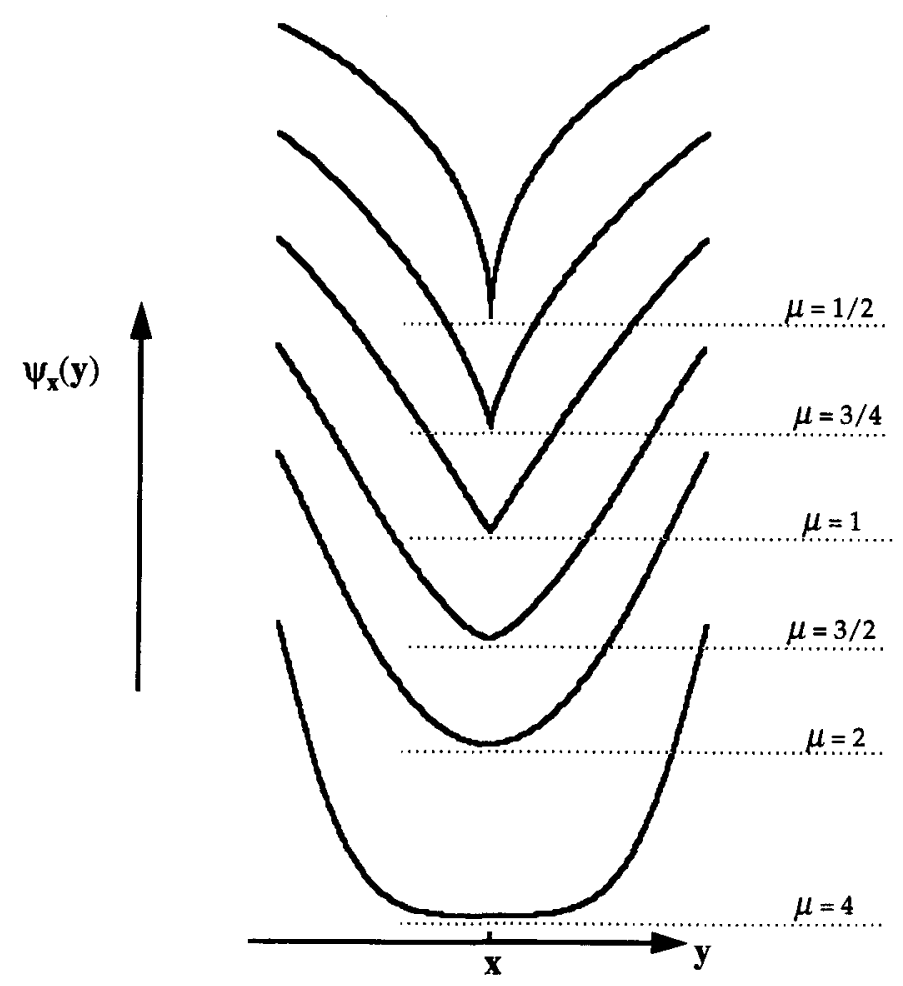

Figure 10. The shapes of unidimensional psychometric functions with different values of $\mu$ in a small vicinity of their global minima. 


$$
x=x(\bar{x}), \bar{x}=\bar{x}(x)
$$

both exist and have continuous positive derivatives. Under these conditions, it is easy to show that

$$
\bar{G}(\bar{a}, \bar{b})=G(a, b)
$$

(see Appendix A2). To avoid redundancy, we do not analyze here other important mathematical properties of the Fechner metric function and the Fechnerian distance. This analysis is presented in the next section, in the context of multidimensional stimulus spaces.

\section{Mathematical Foundations of Fechnerian Metric}

We begin now the construction of a general theory of Fechnerian scaling, for arbitrary (generally multidimensional) continuous stimulus spaces. The elements of such a space and associated $n$-dimensional vectors are denoted by boldface letters ( $\mathbf{x}, \mathbf{y}, \mathbf{u}$, etc.); these vectors' components are denoted as, $\left(x^{1}, \ldots, x^{n}\right),\left(y^{1}, \ldots, y^{n}\right)$, $\left(u^{1}, \ldots u^{n}\right)$, and so on. The superscripts and subscripts $\left(x^{i}, g_{i j}\right.$, etc. $)$ are used to follow the traditional contravariantcovariant conventions of differential geometry, but we utilize no rules or terms of tensor algebra (such as Einstein's summation convention). The context should prevent one from confusing superscripts with exponents. We explain all mathematical terms we introduce if they fall beyond the scope of standard multivariate calculus.

3.1. Psychometric functions. Consider an open space $M^{(n)}$ of $n$-dimensional stimuli $\mathbf{x}=\left(x^{1}, \ldots, x^{n}\right)$. Let any stimulus $\mathbf{x}$ be associated with a psychometric function $\psi_{\mathbf{x}}(\mathbf{y})$ in a canonical form,

$$
\psi_{\mathbf{x}}(\mathbf{y})=\operatorname{Prob}\{\text { “y is different from } \mathbf{x} "\} .
$$

This function is assumed to achieve a global minimum at some point $\mathbf{x}^{*}$ and be defined at least in a sufficiently small open vicinity of this point. Without loss of generality, it can be posited that $\mathbf{x}^{*}=\mathbf{x}$ (see Figure 3 ) - that is,

$$
\psi_{\mathbf{x}}(\mathbf{x})=\min _{\mathbf{y}}\left\{\psi_{\mathbf{x}}(\mathbf{y})\right\}
$$
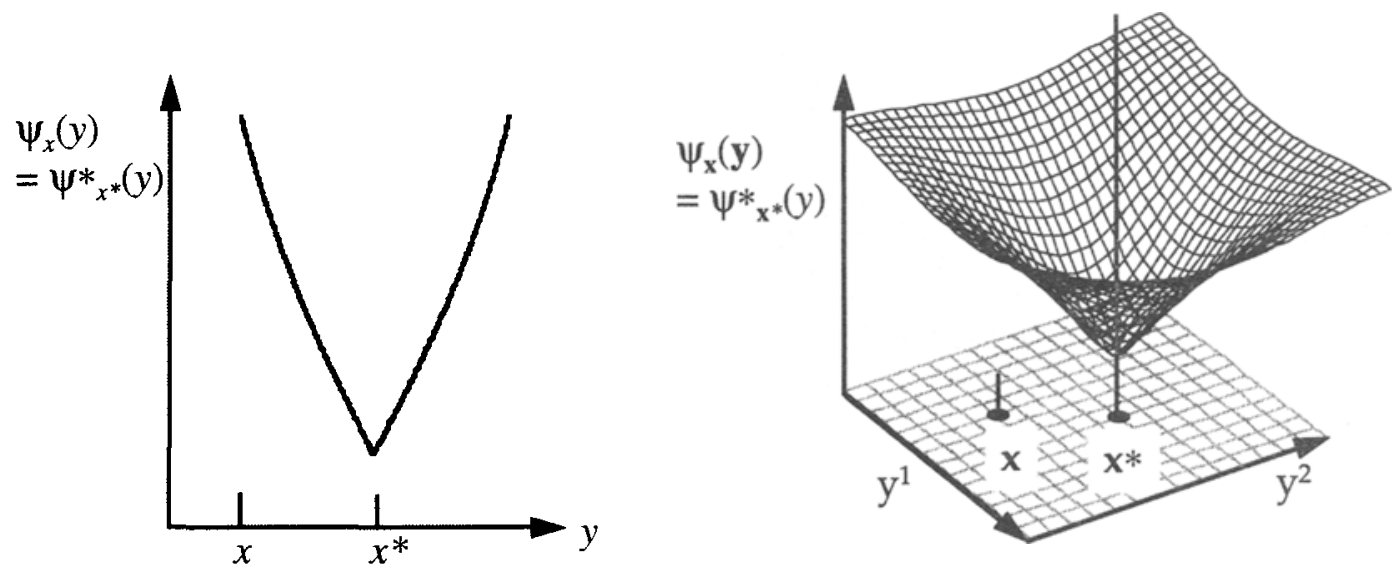

If there is a systematic bias ("constant error"; see Figure 11), then, as in Sections $2.2-2.3$, we assume that $\mathbf{x}^{*}(\mathbf{x})$ is one-to-one, redefine the psychometric function as

$$
\psi^{*}{ }^{*}(\mathbf{y})=\psi_{\mathbf{x}}(\mathbf{y})
$$

and then return to the original notation by dropping the asterisks.

It is often natural to assume, in addition, that the psychometric functions are symmetrical:

$$
\psi_{\mathbf{x}}(\mathbf{y})=\psi_{\mathbf{y}}(\mathbf{x})
$$

We need not, however, invoke this property in this paper (see Section 1.2, d).

3.2. Ray-differentials and ray-derivatives. A ray initiated at $\mathbf{y}$ is defined as $\mathbf{y}+\mathbf{u} s$, where $\mathbf{u}$ is some nonzero vector $\left(u^{1}, \ldots, u^{n}\right)$, and $s$ assumes all real nonnegative values (see Figure 4). The ray-differential of $\psi_{x}(\mathbf{y})$ at $\mathbf{y}$ along $\mathbf{u}$ is defined as

$$
\psi_{\mathbf{x}}(\mathbf{y}+\mathbf{u} s)-\psi_{\mathbf{x}}(\mathbf{y}), s \rightarrow 0+
$$

We are only interested in the ray-differentials at the minimum of the psychometric function, $\mathbf{y}=\mathbf{x}$,

$$
\psi_{\mathbf{x}}(\mathbf{x}+\mathbf{u} s)-\psi_{\mathbf{x}}(\mathbf{x}), s \rightarrow 0+.
$$

Obviously, this difference is positive for all nonzero $s$. Since, for any given $\mathbf{x}$ and $\mathbf{u}$, this difference is an increasing function of $s$ (in a right-hand vicinity of $s=0$ ), there should exist an increasing transformation $\Phi$ vanishing at zero, such that

$$
\Phi\left[\psi_{\mathbf{x}}(\mathbf{x}+\mathbf{u} s)-\psi_{\mathbf{x}}(\mathbf{x})\right]=O(s), s \rightarrow 0+.
$$

In accordance with Section 2.2, we say that the two positive differentials above are comeasurable. It is important to observe that the transformation $\Phi$ is defined asymptotically uniquely in the vicinity of zero: If the comeasurability statement above holds, then it also holds for another transformation $\Phi^{*}$ if and only if

$$
\lim _{a \rightarrow 0+} \frac{\Phi^{*}(a)}{\Phi(a)}=k, 0<k<\infty .
$$

Figure 11. Psychometric functions $\psi_{\mathrm{x}}(\mathrm{y})$ whose global minima at $\mathrm{x}^{*}$ are shifted from the positions of reference stimuli, x. The relabeling procedure $\psi^{*}{ }^{\star}(y)=\psi_{x}(y)$ amounts to viewing the positions of global minima as new reference stimuli. 
Using the same reasoning as in Section 2.2, without much loss of generality, $\Phi$ can be chosen to be a power function, and this is what we assume throughout most of this paper. That is,

(i) for all $\mathbf{x} \in M^{(n)}$ and all nonzero direction vectors $\mathbf{u}$, one can find a positive real $\mu$ such that

$$
\psi_{\mathbf{x}}(\mathbf{x}+\mathbf{u} s)-\psi_{\mathbf{x}}(\mathbf{x})=O\left(\mathrm{~s}^{\mu}\right), s \rightarrow 0+,
$$

which can also be written as

$$
\sqrt[4]{\psi_{\mathbf{x}}(\mathbf{x}+\mathbf{u} s)-\psi_{\mathbf{x}}(\mathbf{x})}=O(s), s \rightarrow 0+
$$

or

$$
0<\lim _{s \rightarrow 0+} \frac{\sqrt[\mu]{\psi_{\mathbf{x}}(\mathbf{x}+\mathbf{u} s)-\psi_{\mathbf{x}}(\mathbf{x})}}{s}<\infty .
$$

It is easy to see that there cannot be more than one $\mu$ satisfying this requirement: If the double-inequality above holds, then

$$
\lim _{s \rightarrow 0+} \frac{\sqrt[\kappa]{\psi_{\mathbf{x}}(\mathbf{x}+\mathbf{u} s)-\psi_{\mathbf{x}}(\mathbf{x})}}{s}=\left\{\begin{array}{l}
0, \text { for } \kappa<\mu \\
\infty, \text { for } \kappa>\mu
\end{array}\right\} .
$$

We further assume that

(ii) the value of $\mu$ is the same for all $\mathbf{x} \in M^{(n)}$ and all nonzero direction vectors $\mathbf{u}$. We refer to $\mu$ as the psychometric order of the stimulus space endowed with the psychometric functions $\psi_{\mathbf{x}}(\mathbf{y})$,

$$
\left\langle\mathbf{x}, \psi_{\mathbf{x}}(\mathbf{y})\right\rangle_{\mathbf{x} \in M^{(n)}},
$$

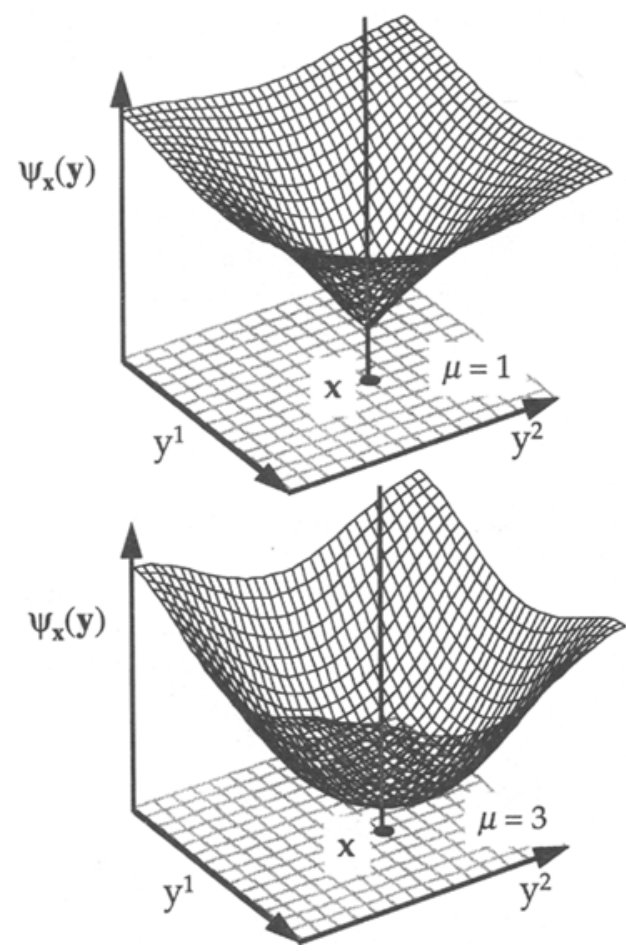

and we denote this structure as $M^{(n, \mu)}$ (see Figures 10 and 12). By abuse of language, we may also refer to $\mu$ as the psychometric order of the set of the psychometric functions $\psi_{\mathbf{x}}(\mathbf{y})$ (indexed by $\mathbf{x}$ ). As mentioned in Section 2.2 and also discussed in Section 7.1, the assumption that a single transformation can be applied to all stimuli and all directions is by far more restrictive and more critical for this paper than the assumption that this transformation is a power function. (In reference to Figure 12, the single- $\mu$ requirement implies that a stimulus space cannot simultaneously contain, say, the cusped psychometric function $\mu=1$ and the flat-bottom psychometric function $\mu=4$.)

Finally, we assume that

(iii) the ray-differential $\psi_{\mathbf{x}}(\mathbf{x}+\mathbf{u} s)-\psi_{\mathbf{x}}(\mathbf{x})$ is symmetri$\mathrm{cal}$ with respect to the sense of any given direction vector:

$$
\lim _{s \rightarrow 0+} \frac{\psi_{\mathbf{x}}(\mathbf{x}+\mathbf{u} s)-\psi_{\mathbf{x}}(\mathbf{x})}{\psi_{\mathbf{x}}(\mathbf{x}-\mathbf{u} s)-\psi_{\mathbf{x}}(\mathbf{x})}=1
$$

This is a generalization of the odd-symmetry assumption made in Section 2.2 and the even-symmetry assumption made in Section 2.3. This property is convenient and reasonable to assume, and it is assumed throughout most of this paper. In Section 7.2, however, we show that it is not essential for the theory, and simple modifications of the Fechnerian metric can be constructed without it.

The equation

$$
\sqrt[\mu]{\psi_{\mathbf{x}}(\mathbf{x}+\mathbf{u} s)-\psi_{\mathbf{x}}(\mathbf{x})}=O(s), s \rightarrow 0+
$$

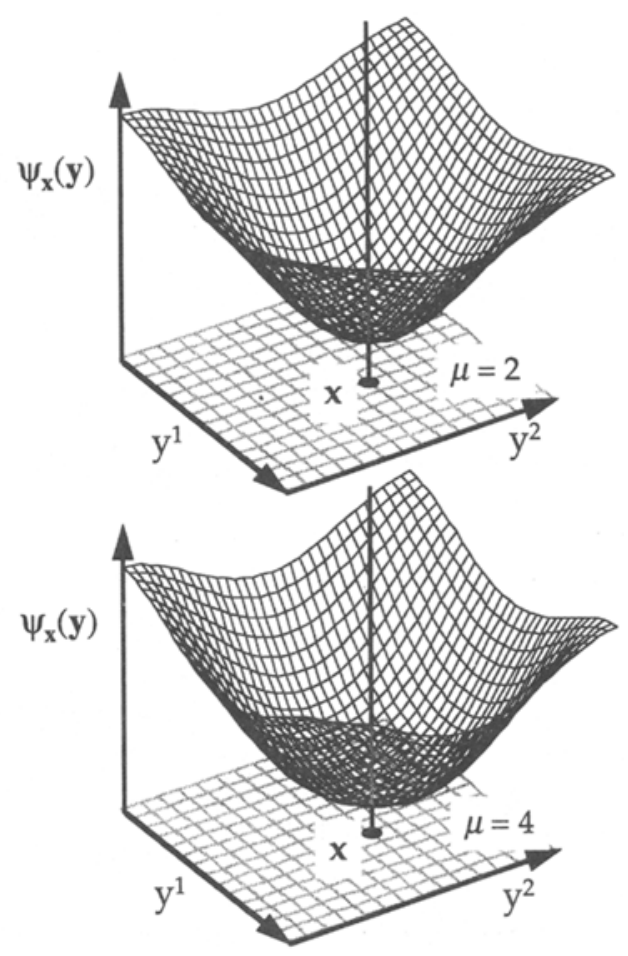

Figure 12. The shapes of two-dimensional psychometric functions with different values of $\mu$ in a small vicinity of their global minima. 
is central for the theory of Fechnerian scaling. It can be interpreted as saying that an infinitesimally small dissimilarity between any two points

$$
\mathbf{x}, \mathbf{x}+\mathbf{u} d s,
$$

can be measured by (as it is comeasurable with) the corresponding infinitesimally small difference

$$
\sqrt[\mu]{\psi_{\mathbf{x}}(\mathbf{x}+\mathbf{u} d s)-\psi_{\mathbf{x}}(\mathbf{x})}
$$

The latter can serve, therefore, as a psychometric distance between $\mathbf{x}$ and $\mathbf{x}+\mathbf{u} d s$. It is important to observe, however, that the latter statement also holds, by precisely the same logic, for the infinitesimal

$$
k \sqrt[\mu]{\psi_{\mathbf{x}}(\mathbf{x}+\mathbf{u} d s)-\psi_{\mathbf{x}}(\mathbf{x})},
$$

with any positive $k$.

3.3. Fechner-Finsler metric function. We call the function

$$
F(\mathbf{x}, \mathbf{u})=\lim _{s \rightarrow 0+} \frac{\sqrt[\mu]{\psi_{\mathbf{x}}(\mathbf{x}+\mathbf{u} s)-\psi_{\mathbf{x}}(\mathbf{x})}}{s}
$$

the (Fechner-Finsler) ${ }^{5}$ metric function associated with $M^{(n, \mu)}$. (For $\mathbf{u}=\mathbf{0}$, the value of the metric function is zero.) The postulated properties of the psychometric functions guarantee that this function is well defined for all stimuli $\mathbf{x}$ and all nonzero direction vectors $\mathbf{u}$ in $M^{(n, \mu)}$.

Due to the concluding statement of the previous section, the Fechner-Finsler metric function is only unique up to positive similarity - that is, it can also be defined as

$$
\lim _{s \rightarrow 0+} \frac{k \sqrt[\mu]{\psi_{\mathbf{x}}(\mathbf{x}+\mathbf{u} s)-\psi_{\mathbf{x}}(\mathbf{x})}}{s},
$$

for any positive $k$. We set $k=1$ for definiteness.

Often, it is convenient to express the metric function as a ray-derivative of the standardized psychometric function

$$
\Psi_{\mathbf{x}}(\mathbf{y})=\sqrt[\mu]{\psi_{\mathbf{x}}(\mathbf{y})-\psi_{\mathbf{x}}(\mathbf{x})}
$$

Obviously, this function has the minimum value of zero achieved at $\mathbf{y}=\mathbf{x}$. The ray-derivative of $\Psi_{\mathbf{x}}(\mathbf{y})$ at $\mathbf{y}$ along $\mathbf{u}$ is defined as

$$
\left.\frac{d \Psi_{\mathbf{x}}(\mathbf{y}+\mathbf{u} s)}{d s}\right|_{s=0+},
$$

and it is easy to observe that

$$
F(\mathbf{x}, \mathbf{u})=\left.\frac{d \Psi_{\mathbf{x}}(\mathbf{x}+\mathbf{u} s)}{d s}\right|_{s=0+} .
$$

3.4. Properties of the metric function. In the following, it is assumed that the Fechner-Finsler metric function $F(\mathbf{x}, \mathbf{u})$ is sufficiently smooth in $\mathbf{u}$ and $\mathbf{x}$. For our purposes, it is sufficient to assume that, for all nonzero directions, $F(\mathbf{x}, \mathbf{u})$ is $C^{2}$ in all arguments (Carathéodory, 1982). ${ }^{6}$ In accordance with Rund's (1959) monograph, however, to connect the theory of Fechnerian scaling with a developed version of the Finslerian geometry (which is not critical in the present context), one has to assume a significantly higher degree of smoothness (e.g., $C^{5}$ in all arguments).

The Fechner-Finsler metric function has the following properties that follow from the postulated properties of the psychometric functions and the definition of the metric function.

(FF1) Strict positivity: $F(\mathbf{x}, \mathbf{u})>0$ for all $\mathbf{x}$ and $\mathbf{u} \neq \mathbf{0}$. This clearly follows from the fact that $\psi_{\mathbf{x}}(\mathbf{x})$ is the minimum of the psychometric function.

(FF2) Symmetry: $F(\mathbf{x},-\mathbf{u})=F(\mathbf{x}, \mathbf{u})$ for all $\mathbf{x}, \mathbf{u}$. This follows from the symmetry requirement (iii) of Section 3.2 .

(FF3) Euler homogeneity of the first order: $F(\mathbf{x}, a \mathbf{u})=$ $a F(\mathbf{x}, \mathbf{u})$ for all $\mathbf{x}, \mathbf{u}$, and $a \geq 0$. (See Appendix A3.) Note that Properties FF2 and FF3 can be combined into

$$
F(\mathbf{x}, a \mathbf{u})=|a| F(\mathbf{x}, \mathbf{u}) .
$$

(FF4) Invariance: The metric function is invariant with respect to all diffeomorphic transformations of the stimulus space, provided that the directions transform as contravariant vectors. This property may appear to be of a somewhat technical nature, but it is critical for justifying the notion that Fechnerian distances among stimuli in $M^{(n, \mu)}$ do not depend on their physical measurements (Sections 3.5-3.6). Consider some diffeomorphic transformations of coordinates,

$$
x^{i}=x^{i}\left(\bar{x}^{1}, \ldots, \bar{x}^{n}\right), i=1, \ldots, n .
$$

Direction vectors are said to transform as contravariant tensors if

$$
u^{i}=\sum_{j=1}^{n} \frac{\partial x^{i}}{\partial \bar{x}^{j}} \bar{u}^{i}, i=1, \ldots, n .
$$

It is easy to verify that with this correspondence between $(\mathbf{x}, \mathbf{u})$ and $(\overline{\mathbf{x}}, \overline{\mathbf{u}})$,

$$
\begin{aligned}
\bar{\psi}_{\overline{\mathbf{x}}}(\overline{\mathbf{x}}+\overline{\mathbf{u}} d s) & -\bar{\psi}_{\overline{\mathbf{x}}}(\overline{\mathbf{x}})=\psi_{\mathbf{x}}(\mathbf{x}(\overline{\mathbf{x}}+\overline{\mathbf{u}} d s)) \\
& -\psi_{\mathbf{x}}(\mathbf{x})=\psi_{\mathbf{x}}(\mathbf{x}+\mathbf{u} d s)-\psi_{\mathbf{x}}(\mathbf{x}),
\end{aligned}
$$

because of which

$$
F(\mathbf{x}, \mathbf{u})=\bar{F}(\overline{\mathbf{x}}, \overline{\mathbf{u}}) .
$$

(FF5) Neighborhood positivity: For any $\rho>0$, the quantity

$$
\varepsilon_{\rho}(\mathbf{x})=\inf _{|\mathbf{y}-\mathbf{x}| \leq \rho}\left\{\inf _{|\mathbf{u}|=1}[F(\mathbf{y}, \mathbf{u})]\right\}
$$

is positive (see Appendix A4). This property is critical for the proof that the Fechnerian distances defined in Section 3.6 do form a metric.

Properties FF 1-FF5 are sufficient to construct the Fechnerian metric. We need, however, one additional as- 
sumption in order to connect this metric with the concept of a geodesic line (Section 3.9).

$\left(\mathrm{FF}^{*}\right)$ Positive regularity: The quantities

$$
g_{i j}(\mathbf{x}, \mathbf{u}) \equiv \frac{1}{2} \frac{\partial^{2} F(\mathbf{x}, \mathbf{u})^{2}}{\partial u^{i} \partial u^{j}}, i, j=1, \ldots, n
$$

form a positive-definite matrix.

The positive-definiteness means that

$$
\sum_{i=1}^{n} \sum_{j=1}^{n} g_{i j}(\mathbf{x}, \mathbf{u}) \zeta^{i} \zeta^{j}>0
$$

unless $\zeta^{1}=\ldots=\zeta^{n}=0$. The matrix of $g_{i j}(\mathbf{x}, \mathbf{u})$ values is called the (Finslerian) metric tensor associated with $F(\mathbf{x}, \mathbf{u})$. The positive regular metric tensor is called Riemannian if $g_{i j}(\mathbf{x}, \mathbf{u})$ does not depend on $\mathbf{u}$ :

$$
g_{i j}(\mathbf{x}, \mathbf{u}) \equiv g_{i j}(\mathbf{x}), i, j=1, \ldots, n .
$$

An important identity that follows from the Euler homogeneity of the metric function is

$$
F(\mathbf{x}, \mathbf{u})^{2}=\sum_{i=1}^{n} \sum_{j=1}^{n} g_{i j}(\mathbf{x}, \mathbf{u}) u^{i} u^{j},
$$

which is the Finslerian generalization of the familiar Riemannian identity,

$$
F(\mathbf{x}, \mathbf{u})^{2}=\sum_{i=1}^{n} \sum_{j=1}^{n} g_{i j}(\mathbf{x}) u^{i} u^{j}
$$

(This identity is commonly written in the form

$$
d S^{2}=\sum_{i=1}^{n} \sum_{j=1}^{n} g_{i j}(\mathbf{x}) d x^{i} d x^{j},
$$

and $d S$ is referred to as the linear element of the Riemannian geometry.)

3.5. Path length. Consider now two points, $\mathbf{a}$ and $\mathbf{b}$, in the stimulus space, and some piecewise continuously differentiable path $\mathbf{x}(t)$ connecting them (refer to Figures 5 and 13),

$$
t \in(a, b), \lim _{t \rightarrow a^{+}} \mathbf{x}(t)=\mathbf{a}, \lim _{t \rightarrow b-} \mathbf{x}(t)=\mathbf{b} .
$$

When we wish to make the endpoints explicit, we write $\left.\mathbf{x}(t)={ }_{\mathbf{a}} \mathbf{x}(t)\right)_{\mathbf{b}}$. The psychometric value of the change from $\mathbf{x}(t)$ to $\mathbf{x}(t+d t)$ is computed as

$$
F(\mathbf{x}(t), \dot{\mathbf{x}}(t)) d t,
$$

where $\dot{\mathbf{x}}(t)$, the tangent vector of the path at $\mathbf{x}(t)$, plays the role of $\mathbf{u}$, the direction of change. Integration of the quantity above along the path yields

$$
\mathfrak{I}\left[\mathbf{a}(t)_{\mathbf{b}}\right]=\int_{a}^{b} F(\mathbf{x}(t), \dot{\mathbf{x}}(t)) d t .
$$

This integral is well defined and can be interpreted as the (Finslerian) length of the arc $\mathbf{x}(t)$ connecting the points a and $\mathbf{b}$. Observe the following properties:

(I1) $\mathfrak{I}\left[\mathbf{a} x(t)_{\mathbf{b}}\right]>0$ unless $\mathbf{x}(t) \equiv \mathbf{a}=\mathbf{b}$, in which case $\mathfrak{I}\left[\mathbf{a} x(t)_{\mathbf{b}}\right] \stackrel{\mathfrak{a}}{=} 0$.

(I2) $\mathfrak{I}\left[{ }_{\mathbf{a}} \mathbf{x}(t)_{\mathbf{b}}\right]=\mathfrak{I}\left[_{\mathbf{a}} \mathbf{x}[\gamma(\tau)]_{\mathbf{b}}\right]$ under all positive diffeomorphic reparametrizations $t=\gamma(t), \dot{\gamma}(\tau)>0$.

(I3) $\mathfrak{I}\left[\mathbf{a} x(t)_{\mathbf{b}}\right]=\mathfrak{I}\left[_{\mathbf{b}} \mathbf{x}[\gamma(\tau)]_{\mathbf{a}}\right]$ under all negative diffeomorphic reparametrizations $t=\gamma(t), \dot{\gamma}(\tau)<0$.

(I4) $\mathfrak{I}\left[\mathbf{a} x(t)_{\mathbf{b}}\right]$ is invariant under all diffeomorphic reparametrizations of the stimulus space.

The proof of these statements is given in Appendix A5.

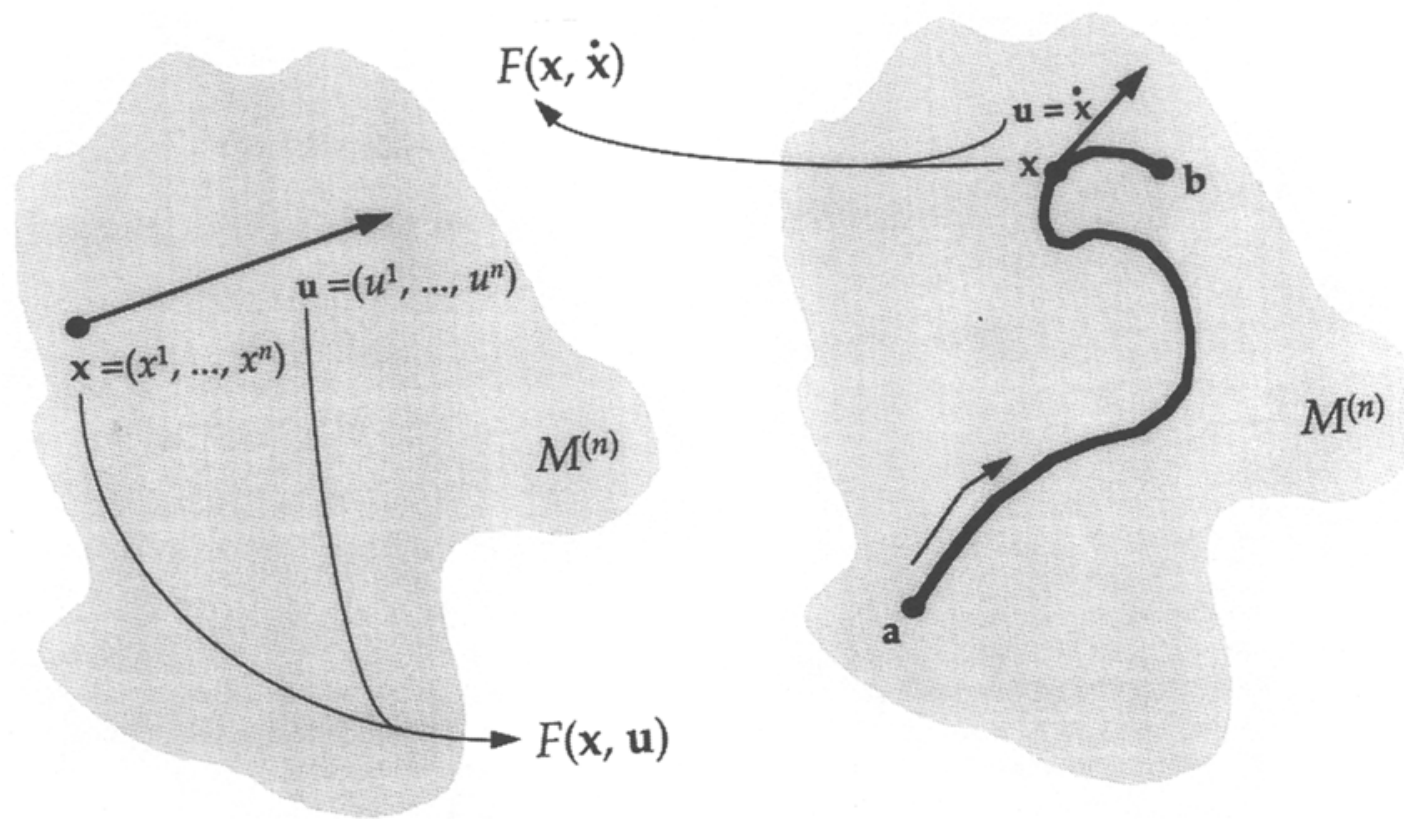

Figure 13. The Fechner-Finsler function $F(x, u)$ is computed for any point stimulus $x$ and direction $u$. If the value of this function is computed at all points $x$ of a (piecewise continuously differentiable) path connecting two points, $a$ and $b$, while the directions $u$ are chosen to coincide with tangents $\dot{x}$ to this path, then the integral of this function is the psychometric length of the path. 
3.6. Fechnerian distance. We define the Fechnerian distance between $\mathbf{a}$ and $\mathbf{b}$ as

$$
G(\mathbf{a}, \mathbf{b})=\inf _{\mathbf{a}(t)_{\mathbf{b}}}\left\{\mathfrak{I}\left[{ }_{\mathbf{a}} \mathbf{x}(t)_{\mathbf{b}}\right]\right\}
$$

where all paths are assumed to be piecewise continuously differentiable. Observe that $\left.\mathfrak{I}_{\mathbf{a}} \mathbf{x}(t)_{\mathbf{b}}\right]$ maps the set of all paths ${ }_{\mathbf{a}} \mathbf{x}(t)_{\mathbf{b}}$ into the set of nonnegative reals, because of which the infimum must exist. The Fechnerian distance has the following properties:

(G1) $G(\mathbf{a}, \mathbf{b})$ is invariant under all diffeomorphic reparametrizations of the paths $\mathbf{a}_{\mathbf{a}} \mathbf{x}(t)_{\mathbf{b}}$.

(G2) $G(\mathbf{a}, \mathbf{b})$ is invariant under all diffeomorphic reparametrizations of the stimulus space.

(G3) $G(\mathbf{a}, \mathbf{b})$ is a distance function on the stimulus space.

(G4) $G(\mathbf{a}, \mathbf{b})$ is unique up to positive similarity.

The first two properties follow directly from the corresponding properties of $\mathfrak{I}[\mathbf{a}(t)$ ] the Fechnerian distance as defined in this section satisfies the following three statements:

$$
\begin{gathered}
G(\mathbf{a}, \mathbf{b})=G(\mathbf{b}, \mathbf{a}) \text { (symmetry) } \\
G(\mathbf{a}, \mathbf{b}) \leq G(\mathbf{a}, \mathbf{c})+G(\mathbf{c}, \mathbf{b}) \text { (triangle inequality) } \\
G(\mathbf{a}, \mathbf{b})=\mathbf{0} \text { iff } \mathbf{a}=\mathbf{b} \text { (zero distance property) }
\end{gathered}
$$

The proof of these statements is given in Appendix A6. Property G4 follows from the fact that the metric function $F(\mathbf{x}, \mathbf{u})$ is unique up to positive similarity.

3.7. Uniqueness of psychometric functions inducing a given metric. Property G4 justifies the following definition: For a given stimulus space, two different sets of psychometric functions, $\psi_{\mathbf{x}}(\mathbf{y})$ and $\bar{\psi}_{\mathbf{x}}(\mathbf{y})$, are called Fechner-equivalent if they induce Fechnerian metrics related as

$$
F(\mathbf{x}, \mathbf{u})=k \bar{F}(\mathbf{x}, \mathbf{u}) .
$$

This equality implies

$$
\frac{\sqrt[\mu]{\bar{\psi}_{\mathbf{x}}(\mathbf{x}+\mathbf{u} s)-\bar{\psi}_{\mathbf{x}}(\mathbf{x})}}{\sqrt[\mu]{\psi_{\mathbf{x}}(\mathbf{x}+\mathbf{u} s)-\psi_{\mathbf{x}}(\mathbf{x})}}=k+o\{1\}, s>0,
$$

where $\mu$ and $\bar{\mu}$ are the (generally different) psychometric orders associated with $\psi_{\mathbf{x}}(\mathbf{y})$ and $\bar{\psi}_{\mathbf{x}}(\mathbf{y})$. By rewriting this as

$$
\begin{aligned}
\bar{\psi}_{\mathbf{x}}(\mathbf{x}+\mathbf{u} s) & -\bar{\psi}_{\mathbf{x}}(\mathbf{x})=k^{\bar{\mu}}\left[\psi_{\mathbf{x}}(\mathbf{x}+\mathbf{u} s)\right. \\
& \left.-\psi_{\mathbf{x}}(\mathbf{x})\right]^{\bar{\mu}}{ }^{\bar{\mu}}+o\left\{s^{\bar{\mu}}\right\}, s>0,
\end{aligned}
$$

and denoting $\bar{\psi}_{\mathbf{x}}(\mathbf{x})=\varphi(\mathbf{x}), \kappa \mu=\bar{\mu}, k^{\bar{\mu}}=K$, we come to the following conclusion: Two sets of psychometric functions, $\psi_{\mathbf{x}}(\mathbf{y})$ and $\bar{\psi}_{\mathbf{x}}(\mathbf{y})$, are Fechner-equivalent if and only if

$$
\begin{aligned}
\bar{\psi}_{\mathbf{x}}(\mathbf{x}+\mathbf{u} s)= & K\left[\psi_{\mathbf{x}}(\mathbf{x}+\mathbf{u} s)-\psi_{\mathbf{x}}(\mathbf{x})\right]^{\kappa} \\
& +\varphi(\mathbf{x})+o\left\{s^{\kappa \mu}\right\}, s>0
\end{aligned}
$$

where $K$ and $\kappa$ are arbitrary positive constants, and $\varphi(\mathbf{x})$ is an arbitrary nonnegative function. This criterion of Fechner-equivalence is utilized in Section 5, in the context of response bias changes.

3.8. Possible relationships between metric functions. It might appear that one could generalize the notion of the Fechner-equivalence by considering the sets of psychometric functions, $\psi_{\mathbf{x}}(\mathbf{y})$ and $\bar{\psi}_{\mathbf{x}}(\mathbf{y})$, whose FechnerFinsler metric functions are related by some arbitrary transformation:

$$
F(\mathbf{x}, \mathbf{u})=f[\bar{F}(\mathbf{x}, \mathbf{u})] .
$$

Such sets of psychometric functions, if they existed, could be termed Fechner-related, even though the Fechnerian distances $G(\mathbf{a}, \mathbf{b})$ and $\bar{G}(\mathbf{a}, \mathbf{b})$ computed from them would not have been generally related by any function.

A simple but remarkable fact is, however, that there can be no "Fechner-relatedness" other than Fechnerequivalence, which means that the transformation $f$ in the equation above cannot be anything but positive similarity:

$$
F(\mathbf{x}, \mathbf{u})=f[\bar{F}(\mathbf{x}, \mathbf{u})] \Rightarrow F(\mathbf{x}, \mathbf{u})=k \bar{F}(\mathbf{x}, \mathbf{u}), k>0 .
$$

The proof of this statement follows directly from the Euler homogeneity property of the metric functions (see Appendix A7).

3.9. Fechnerian geodesics. The construction of the Fechnerian metric $G(\mathbf{a}, \mathbf{b})$ in Section 3.6 does not make use of the positive regularity property of the metric function, one consequence of this being that $G(\mathbf{a}, \mathbf{b})$ need not exist as the length of a path connecting the two points. ${ }^{8}$ If we do take into account the positive regularity, then the theory can be developed further as follows.

A path $\mathbf{x}(t)$ is called stationary if it satisfies the EulerLagrange differential equations

$$
\frac{\partial F(\mathbf{x}(t), \dot{\mathbf{x}}(t))}{\partial x^{i}(t)}-\frac{d}{d t}\left(\frac{\partial F(\mathbf{x}(t), \dot{\mathbf{x}}(t))}{\partial \dot{x}^{i}(t)}\right)=0, i=1, \ldots, n .
$$

A fundamental theorem of variational calculus states that if $\mathbf{x}(t)$ connects $\mathbf{a}$ and $\mathbf{b}$, then these equations are a necessary condition for $\mathbf{x}(t)$ to be the shortest (in the Finslerian sense) path connecting the two points (Carathéodory, 1982; Rund, 1959). In general, however, a stationary path connecting two given points need not exist, and, if it exists, it need not provide a global minimum for length of all allowable paths connecting these points. With the complete set of assumptions specified in Section 3.4, however (including the positive regularity property), the following can be proved (Carathéodory, 1982, ch. 16):

(G5) If the stimulus space, $M^{(n)}$, is bounded (i.e., if $|\mathbf{b}-\mathbf{a}|<\Lambda$ for all $\mathbf{a}, \mathbf{b}$, and some constant $\Lambda$ ), then any two points $\mathbf{a}, \mathbf{b}$ can be connected by at least one stationary line, and all stationary lines connecting these points will have the Finslerian length $G(\mathbf{a}, \mathbf{b})$.

For unbounded stimulus spaces this theorem holds in a weaker version: 
(G6) If the stimulus space contains a point 0 such that

$$
G(\mathbf{x}, \mathbf{0}) \rightarrow \infty \text { as }|\mathbf{x}-\mathbf{0}| \rightarrow \infty,
$$

then any two points $\mathbf{a}, \mathbf{b}$ can be connected by at least one stationary line, and all stationary lines connecting these points will have the Finslerian length $G(\mathbf{a}, \mathbf{b})$.

The meaning of the additional constraint imposed in G5 is that, when considering the paths connecting two given points in order to find the shortest, one can always confine one's attention to those paths that lie within a sufficiently large bounded area containing the two points. This is automatically satisfied if the space itself is bounded, because of which G5 can be viewed as a consequence of G6.

The antecedent of G6 is the only assumption in this paper that does not refer to observable (at least in principle) properties of psychometric functions. It is not, therefore, empirically falsifiable, even in principle. The importance of this fact, however, should not be overestimated. Geodesics are not, strictly speaking, necessary for the construction of a Fechnerian metric, but, even if they were, it seems safe to assume that any stimulus space is bounded: As one moves from a given point in a stimulus space in any direction, it is inevitable that, beyond a certain distance, the stimuli are no longer perceivable. If so, the existence and the desired properties of the geodesics are guaranteed by $\mathrm{G} 5$ alone.

3.10. Perceptual status of geodesics. If the Fechnerian geodesics of a stimulus space can be reconstructed (by computing the Fechner-Finsler functions and solving the Euler-Lagrange differential equations, analytically or numerically), one achieves much more than just the ability to compute Fechnerian distances effectively. A variety of descriptive geometric concepts (not discussed here) then can be invoked to characterize and classify perceptual structures of stimulus spaces. It should be emphasized, however, that our theory does not imply that the geodesics (or other geometric descriptors associated with them) can be in any way perceptually accessible to the observers or directly mappable on the observers' judgments: It is not implied, for instance, that the observers are able to determine whether series of stimuli fall on a single geodesic or judge whether two stimulus changes in the directions tangent to a geodesic are parallel. The theory is based solely on psychometric functions, the only prerequisite ability of the observers being that of discriminating (or confusing) different stimuli.

This distinguishes our approach from the only other theory known to us (Levin, in press) that also aims at generalizing Fechnerian scaling to continuous stimulus spaces of arbitrary dimensionality and arbitrary modality. Levin departs from a generalization of the Riemannian geometry very different from the one our analysis arrives at. This geometric construct (affinely connected spaces with autoparallel curves as generalized geodesics) need not be reviewed here (see, e.g., Kreyszig, 1968, ch. 14). What is important in the present context is that, in order to reconstruct the principal determinants of this construct (the symmetrical connection and the torsion tensor parts of the affine connection), Levin assumes that geodesic paths in a stimulus space can be recognized and compared perceptually. Namely, given a stimulus a and a direction of change $\mathbf{u}$, the observers are assumed to be able to continuously transform a so that the path of the transformation $\mathbf{x}(t)$ forms the geodesic passing through $\mathbf{a}$ in the direction $\mathbf{u}$. Moreover, considering $t$ in $\mathbf{x}(t)$ as physical time, the observers are assumed to be able to perform this transformation at a "constant perceptual speed," which means that the tangents $d \mathbf{x}(t) / d t$ along the path are all of the same subjective length. Finally, the observers are assumed to be able to "review" a finite segment of a geodesic path, say, $\mathbf{a}(t)_{\mathbf{b}}$ leading from $\mathbf{a}$ to $\mathbf{b}$, and given a stimulus c some distance apart from it, to transform this $\mathbf{c}$ along the geodesic running "parallel" to $\mathbf{a}(t)_{\mathbf{b}}$ at the same constant perceptual speed. Although the observers in Levin's experiments do produce stimulus transformations in response to these instructions, it is not obvious to us that they do this in conformity with Levin's geometric interpretation.

In a contradistinction to our approach, Levin (in press) views discriminability as limited by finite JNDs, and he treats them as a nuisance factor completely unrelated to his theory but relegating it to the status of an approximation only. This makes the logic of his approach subject to the same internal inconsistency criticism (recognized in Levin's paper) as the one directed against Fechner's original theory, in its traditional interpretation (Section 2.1).

\section{Special Cases}

4.1. Unidimensional Fechnerian scaling. We show now how the theory of unidimensional scaling developed in Sections 2.2-2.3 is derived as a special case of the general theory presented in Section 3. Recall the definition and the properties of the standardized psychometric function $\Psi_{\mathbf{x}}(\mathbf{y})$ given in Section 3.3. In the unidimensional case, it becomes

$$
\Psi_{x}(y)=\sqrt[\mu]{\psi_{x}(y)-\psi_{x}(x)} .
$$

For any psychometric order $\mu$, we have

$$
F(x, u)=\left.\frac{d \Psi_{x}(x+u s)}{d s}\right|_{s=0+}=\left.|u| \frac{d \Psi_{x}(y)}{d y}\right|_{y=x+},
$$

because of which the metric function can be written as

$$
F(x, u)=\xi(x)|u|, \xi(x)>0 .
$$

The shortest path between $\mathbf{a}=a$ and $\mathbf{b}=b>a$ is, of course,

$$
{ }_{\mathbf{a}} x(t)_{\mathbf{b}}=t, t \in(a, b),
$$

because any other path will have to include the interval $(a, b)$. As a result, the Fechnerian distance here is 


$$
G(a, b)=\int_{a}^{b} \xi(t) d t .
$$

Suppose, for example, that $\mu=1$ and (the infinitesimal version of) Weber's law holds:

$$
\xi(x)=\left.\frac{d \psi_{x}(y)}{d y}\right|_{y=x+}=\frac{k}{x} .
$$

Then,

$$
G(a, b)=k \log \frac{b}{a} .
$$

If $\mu$ is not necessarily 1 , Weber's law has the general form

$$
\left.\frac{d \Psi_{x}(y)}{d y}\right|_{y=x+}=\frac{k}{x},
$$

leading to the same logarithmic Fechner law.

Obviously, one can also consider other differential "laws" (very much like Fechner himself did in his 1860 treatise) to obtain alternatives to the "logarithmic law." For example,

$$
\left.\frac{d \Psi_{x}(y)}{d y}\right|_{y=x+}=\left(\frac{k}{x}\right)^{1-\alpha}, 0<\alpha<1,
$$

leads to

$$
G(a, b)=\text { const } \cdot\left(b^{\alpha}-a^{\alpha}\right),
$$

the "compressive power function" that Krueger (1989) proposes as a solution for the controversy related to competing "psychophysical laws."

4.2. Riemannian metric. Several authors have suggested that the subjective metric of multidimensional continuous stimulus spaces is Riemannian [which means that the metric tensor $g_{i j}(\mathbf{x}, \mathbf{u})$ does not depend on $\left.\mathbf{u}\right]$ and that the formula for the Riemannian linear element (see the end of Section 3.4) can be viewed as a multidimensional generalization of Fechner's differential formula. It is especially apparent in Helmholtz's (1891) analysis of color differences $(n=3)$, where the proposed metric tensor is modeled after the infinitesimal version of Weber's law: Using our present notation,

$$
\left[g_{i j}(\mathbf{x})\right]=\left[\begin{array}{ccc}
\frac{1}{\left(x^{1}\right)^{2}} & 0 & 0 \\
0 & \frac{1}{\left(x^{2}\right)^{2}} & 0 \\
0 & 0 & \frac{1}{\left(x^{3}\right)^{2}}
\end{array}\right],
$$

which corresponds to

$$
F(\mathbf{x}, \mathbf{u})^{2}=\sum_{i=1}^{3} \frac{1}{\left(x^{i}\right)^{2}}\left(u^{i}\right)^{2} .
$$

Schrödinger (1920) proposed to amend this as $\left[g_{i j}(\mathbf{x})\right]=$

$$
\left[\begin{array}{ccc}
\frac{1}{x^{1}\left(x^{1}+x^{2}+x^{3}\right)} & 0 & 0 \\
0 & \frac{1}{x^{2}\left(x^{1}+x^{2}+x^{3}\right)} & 0 \\
0 & 0 & \frac{1}{x^{3}\left(x^{1}+x^{2}+x^{3}\right)}
\end{array}\right],
$$

corresponding to

$$
F(\mathbf{x}, \mathbf{u})^{2}=\sum_{i=1}^{3} \frac{1}{x^{i}\left(x^{1}+x^{2}+x^{3}\right)}\left(u^{i}\right)^{2} .
$$

The contemporary CIE recommendations on the color metric (Robertson, 1978) retain the idea of the Riemannian geometry while replacing theoretical guesses about the form of the metric tensor, such as Helmholtz's and Schrödinger's, with empirically adapted formulas.

Although some empirical work has been done with psychometric functions $\psi_{\mathbf{x}}(\mathbf{y})$ in color spaces (Indow, 1993; Indow \& Morrison, 1991; Indow, Robertson, von Grunau, \& Fielder, 1992; Witt \& Döring, 1983), no attempt is known to us to systematically derive, at least in principle, the postulated Riemannian metric from the shapes of these functions. Nor do we know of an attempt to achieve a sufficient precision in plotting the psychometric functions in a small vicinity of their minima. As explained in Section 7.3, if one bases one's approach on the shape of psychometric functions, there are no compelling reasons for the assumption that the Fechnerian metrics must be Riemannian.

4.3. Radial symmetry. Suppose that, with some parametrization of the stimulus space, the psychometric functions acquire radial symmetry at their minima with respect to a certain norm, not necessarily Euclidean or even quadratic. This means that for all vectors $\boldsymbol{\mu}$ having the same norm, $|\mathbf{u}|=$ const, the expression

$$
F(\mathbf{x}, \mathbf{u})=\left.\frac{d \Psi_{x}(\mathbf{y}+\mathbf{u} s)}{d s}\right|_{s=0+}
$$

is constant in $\mathbf{u}$.

Denoting

$$
\left.\frac{d \Psi_{x}\left(\mathbf{x}+\mathbf{u}^{*} s\right)}{d s}\right|_{s=0+}=\xi(\mathbf{x})
$$

for all unit-norm direction vectors, $\left|\mathbf{u}^{*}\right|=1$, we have

$$
F(\mathbf{x}, \mathbf{u})=\xi(\mathbf{x})|\mathbf{u}|, \xi(\mathbf{x})>0 .
$$

Consequently,

$$
\mathfrak{I}\left[\mathbf{a}(t)_{\mathbf{b}}\right]=\int_{a}^{b} F(\mathbf{x}(t), \dot{\mathbf{x}}(t)) d t=\int_{a}^{b} \xi(\mathbf{x}(t))|\dot{\mathbf{x}}(t)| d t,
$$


and the Fechnerian distance is computed as the infimum of these integrals.

The Finsler metric tensor in this case is

$$
\begin{aligned}
g_{i j}(\mathbf{x}, \mathbf{u}) & =\frac{1}{2} \frac{\partial^{2} F(\mathbf{x}, \mathbf{u})^{2}}{\partial u^{i} \partial u^{j}} \\
& =\xi(\mathbf{x})^{2} \frac{\partial^{2}|\mathbf{u}|^{2}}{\partial u^{i} \partial u^{j}}=\gamma_{i j}(\mathbf{u}) \xi(\mathbf{x})^{2},
\end{aligned}
$$

which is not generally a Riemannian tensor since it depends on the direction vector $\mathbf{u}$.

4.4. Minkowskian metric. A metric is called Minkowskian if the metric function $F(\mathbf{x}, \mathbf{u})$ is location-independent,

$$
F(\mathbf{x}, \mathbf{u})=F(\mathbf{u}) \text {. }
$$

This is certainly the simplest special case of the Finslerian metric. It corresponds to the situation when all psychometric functions $\psi_{\mathbf{x}}(y)$ are essentially identical in the vicinity of their minima. In this situation, the EulerLagrange differential equations acquire the form

$$
\frac{\partial F(\dot{\mathbf{x}}(t))}{\partial \dot{x}^{i}(t)}=c_{i}, i=1, \ldots, n,
$$

where $c_{i}$ are arbitrary constants. The solution of these equations is the straight line passing through two given points,

$$
\mathbf{x}(t)=\mathbf{a}+(\mathbf{b}-\mathbf{a}) t,
$$

and the Fechnerian distance is simply

$$
G(\mathbf{a}, \mathbf{b})=F(\mathbf{b}-\mathbf{a}) \text {. }
$$

\section{Discriminability Versus Response Bias}

A variety of experimental manipulations affect psychometric functions in such a way as to change the Fechnerian distances computed from them. For some manipulations (such as prolonged preexposure to a particular stimulus), the changes in the Fechnerian metric are interpretable as reflecting those in the "true perceptual discriminability" of the stimuli, in some intuitive sense. It has long been recognized in psychophysics, however, that psychometric functions obtained by means of a given judgment scheme generally reflect not only the true discriminability but also the decision maker's differential predispositions toward the responses to be chosen among. In the present context, this means that the psychometric functions can be presented as

$$
\operatorname{Prob}\{\text { " } \mathbf{y} \text { is different from } \mathbf{x} "\}=\psi_{\mathbf{x}, \boldsymbol{\theta}}(\mathbf{y}),
$$

where $\theta$ is a vector of response bias parameters that, by the virtue of being so labeled, are believed to have nothing to do with the true perceptual discriminability of $\mathbf{y}$ from $\mathbf{x}$.

As an example, in a broad class of models known as signal detectability theories (for unidimensional stimulus continua), the response bias is a real-valued $\theta$ (the "criterion") in the representation

$$
\psi_{x, \theta}(y)=T\left[\delta_{x}(y)+\theta\right],
$$

where $T$ is a strictly increasing distribution function (e.g., the unit normal integral in the most commonly used version), and $\delta_{x}(y)$ is a measure of discriminability of $y$ from $x$. If one subscribes to such a model, then one might find it undesirable that the Fechnerian metric may change as a result of changes in the response bias parameters alone.

We consider now two different, from the standpoint of Fechnerian scaling, approaches to the definition of the dichotomy of discriminability versus response bias. According to one of them, which we call Fechner-independent, this dichotomy can be defined in a variety of ways by models completely unrelated to Fechnerian scaling. The goal is, therefore, for any such a model, to construct a Fechnerian metric that is bias-invariant in the corresponding sense. This goal is achieved with no major complications if the model's discriminability function $\delta_{\mathbf{x}}(\mathbf{y})$ is unidimensional and if, for at least one fixed value of the response bias $\boldsymbol{\theta}$, the model provides a way of computing the difference of the discriminability values

$$
\delta_{\mathbf{x}}(\mathbf{y})-\delta_{\mathbf{x}}(\mathbf{x})
$$

from a complete set of psychometric functions $\psi_{\mathbf{x}, \boldsymbol{\theta}}(\mathbf{y})$ :

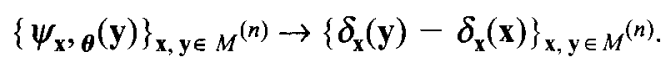

The applicability of such a computation is predicated on the assumption that the value of $\boldsymbol{\theta}$ remains fixed under specific experimental conditions.

It seems safe to assume, for a reasonable response bias model, that the difference $\delta_{\mathbf{x}}(\mathbf{y})-\delta_{\mathbf{x}}(\mathbf{x})$ possesses all the properties postulated in Sections 3.1-3.4 for $\psi_{\mathbf{x}}(\mathbf{y})-$ $\psi_{\mathbf{x}}(\mathbf{x})$. Because of this, one can define a "true" FechnerFinsler metric function,

$$
F_{\delta}(\mathbf{x}, \mathbf{u})=\lim _{s \rightarrow 0+} \frac{\sqrt[\mu]{\delta_{x}(\mathbf{x}+\mathbf{u} s)-\delta_{\mathbf{x}}(\mathbf{x})}}{s},
$$

and construct the "true" Fechnerian metric $G_{\delta}(\mathbf{a}, \mathbf{b})$ in precisely the same way as before.

Thus, in the example with the unidimensional signal detectability theories, we have

$$
\delta_{x}(y)-\delta_{x}(x)=T^{-1}\left[\psi_{x, \theta}(y)\right]-T^{-1}\left[\psi_{x, \theta}(x)\right],
$$

which is obviously independent of $\theta$ (if $T$ is the unit normal integral, this measure is the conventional $d^{\prime}$; Creelman, 1967, proposed to use $d^{\prime}$ for Fechnerian scaling in a manner very similar to the theory presented in Section 2). It is easy to check that, for a broad class of wellbehaved transformations $T$, if the properties postulated in Sections 3.1-3.4 hold for $\psi_{x, \theta}(y)-\psi_{x, \theta}(x)$, then they should also hold for $\delta_{x}(y)-\delta_{x}(x)$.

According to the second, Fechner-dependent, approach to the dichotomy of discriminability versus response bias, the notion of discriminability is simply identified with that of the Fechnerian metric: If the latter changes, then 
the discriminability changes by definition, whatever the manipulations that cause the change, or its interpretation in terms of the underlying psychological processes. The Fechnerian metric, according to such a position, reflects the observable differences in response probabilities associated with different pairs of stimuli, rather than unobservable "perceptual distances." Thus, all the Fechnerian distances legitimately collapse to zero if the response " $y$ differs from $\mathbf{x}$ " is given with the same probability to all pairs of stimuli, because no two stimuli in such a situation are judged to be more different from each other than either of them is from itself.

This approach does not dispense with the notion of a response bias altogether, but it relegates this notion to only those transformations of the psychometric functions that preserve their Fechner-equivalence, in the sense of Section 3.7:

$$
\begin{aligned}
& \psi_{\mathbf{x}}, \theta^{*}(\mathbf{x}+\mathbf{u} s)=K\left[\psi_{\mathbf{x}}, \boldsymbol{\theta}(\mathbf{x}+\mathbf{u} s)\right. \\
& \left.-\psi_{\mathbf{x}}, \boldsymbol{\theta}(\mathbf{x})\right]^{\kappa}+\varphi_{\boldsymbol{\theta}, \boldsymbol{\theta}^{*}}(\mathbf{x})+o\left\{s^{\kappa \mu}\right\}, s>0,
\end{aligned}
$$

for any two values of the response bias parameters, $\boldsymbol{\theta}$ and $\boldsymbol{\theta}^{*}$. This expression can be further simplified if one assumes, as seems natural to do, that response bias changes preserve the psychometric order $\mu$ (i.e., $\kappa=1$ ):

$$
\psi_{\mathbf{x}, \boldsymbol{\theta}^{*}}(\mathbf{x}+\mathbf{u} s)=K \psi_{\mathbf{x}, \boldsymbol{\theta}}(\mathbf{x}+\mathbf{u} s)+\sigma_{\boldsymbol{\theta}, \boldsymbol{\theta}^{*}}(\mathbf{x})+o\left\{s^{\mu}\right\}, s>0,
$$

where $\sigma_{\boldsymbol{\theta}, \boldsymbol{\theta}^{*}}(\mathbf{x})$ is an arbitrary nonnegative function.

An example of a response bias model that satisfies this requirement is

$$
\psi_{\mathbf{x}}, \boldsymbol{\theta}(\mathbf{y})=T_{\boldsymbol{\theta}}\left(\delta_{\mathbf{x}}(\mathbf{y})\right)+\sigma_{\boldsymbol{\theta}}(\mathbf{x}),
$$

where $\delta_{\mathbf{x}}(\mathbf{y})$ has the properties postulated in Sections 3.1-3.4, with the additional constraint

$$
\delta_{\mathbf{x}}(\mathbf{x})=0,
$$

$T_{\boldsymbol{\theta}}$ are increasing transformations vanishing at zero and such that

$$
0<\left.\frac{d T_{\theta}(a)}{d a}\right|_{a=0+}<\infty,
$$

while $\sigma_{\theta}(\mathbf{x})$ is an arbitrary nonnegative function.

Given the apparently restrictive character of the Fechnerdependent approach, it is a surprising and remarkable fact that the class of the response bias models consistent with it is very broad: Virtually any conceivable model with a unidimensional discriminability function $\delta_{\mathbf{x}}(\mathbf{y})$ can be presented as a special case of the example just given. In other words, virtually all conceivable models with a unidimensional discriminability function predict that the classes of psychometric functions corresponding to different response bias values are Fechner-equivalent. (The most familiar example of a response bias model that does not have a unidimensional discriminability function is the normal-normal signal detectability model with unequal variance values: There, the discriminability function is two-dimensional, one component being the difference of the means, and another being the ratio of the variances.)
Due to the remarkable fact about the Fechner-Finsler metric function established in Section 3.8, there can be no compromise between the Fechner-independent and Fechner-dependent approaches to the dichotomy of discriminability versus response bias. If Fechner-Finsler metric functions $F_{\boldsymbol{\theta}}(\mathbf{x}, \mathbf{u})$ and $F_{\theta^{\star}}(\mathbf{x}, \mathbf{u})$ corresponding to two different values of the response bias parameters are related by any transformation at all,

$$
F_{\boldsymbol{\theta}^{*}}(\mathbf{x}, \mathbf{u})=f_{\boldsymbol{\theta}^{*}},{ }_{\theta}\left[F_{\theta}(\mathbf{x}, \mathbf{u})\right],
$$

then this transformation must be positive similarity:

$$
\begin{gathered}
F_{\boldsymbol{\theta}^{\star}}(\mathbf{x}, \mathbf{u})=f_{\boldsymbol{\theta}^{\star}, \boldsymbol{\theta}}\left[F_{\boldsymbol{\theta}}(\mathbf{x}, \mathbf{u})\right] \Rightarrow \\
F_{\boldsymbol{\theta}^{\star}}(\mathbf{x}, \mathbf{u})=c_{\boldsymbol{\theta}^{\star}, \boldsymbol{\theta}} F_{\boldsymbol{\theta}}(\mathbf{x}, \mathbf{u}), c_{\boldsymbol{\theta}^{*}, \boldsymbol{\theta}}>0 .
\end{gathered}
$$

\section{Subjectively Unidimensional \\ Psychometric Functions}

6.1. Theory. Here, we consider the extension of our theory of Fechnerian scaling to psychometric functions that represent discriminability of multidimensional stimuli with respect to a unidimensional subjective attribute, ८. A classical example (Fletcher \& Munson, 1933) is the discriminability of loudness levels $(\ell)$ of pure tones that differ in both intensity and frequency $\left(y^{1}, y^{2}\right)$. Keeping this example in mind enables us to address (in the next section, see also Section 1.1) what is arguably the sharpest available experimental critique of Fechner's original theory.

As in the case of a unidimensional physical continuum, the psychometric functions for the subjectively unidimensional discriminations can be obtained both in a canonical form,

$$
\begin{aligned}
& \psi_{\mathbf{x}}(\mathbf{y})=\operatorname{Prob}\{\text { "the amount of } \ell \text { in } \mathbf{y} \\
& \qquad \text { is different from the amount of } \ell \text { in } \mathbf{x} "\},
\end{aligned}
$$

and in noncanonical forms, such as

$$
\gamma_{\mathbf{x}}(\mathbf{y})=\operatorname{Prob}\{\text { " the amount of } \ell \text { in } \mathbf{y}
$$

is greater than the amount of $\ell$ in $\left.x^{\prime \prime}\right\}$.

In the latter case, we employ the same mirror-reflection procedure as in Section 2.3 to bring $\gamma_{\mathbf{x}}(\mathbf{y})$ to a canonical form:

$$
\psi_{\mathbf{x}}(\mathbf{y})=\left\{\begin{array}{ll}
\gamma_{\mathbf{x}}(\mathbf{y}) & \text { if } \gamma_{x}(y) \geq 1 / 2 \\
1-\gamma_{\mathbf{x}}(\mathbf{y}) & \text { if } \gamma_{x}(y)<1 / 2
\end{array} .\right.
$$

As in Section 2.3, we do not assume that different judgment schemes should yield the same Fechnerian metric, but we assume only that the computational procedure can be made the same in all cases by presenting the psychometric functions in the form of $\psi_{\mathbf{x}}(\mathbf{y})$.

The crucial modification that has to be made in the theory when applied to subjectively unidimensional discriminations is that the Fechnerian distances here are defined between stimulus classes of $\ell$-equivalence (within each of which the stimuli have the same magnitude of $\ell$ ) rather than between individual stimuli, as in the theory 


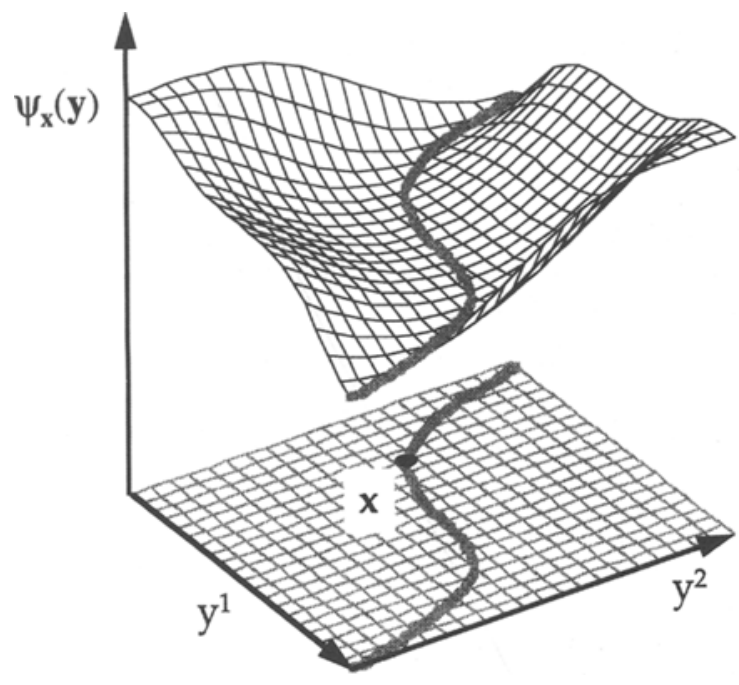

Figure 14. Possible appearance of a two-dimensional psychometric function $\psi_{\mathbf{x}}(\mathbf{y})=$ Prob; " $y$ is different from $x$ with respect to $\ell$ " $\}$; the minima of $\psi_{\mathrm{x}}(\mathrm{y})$ are assumed to form a line whose projection on the stimulus plane is also a line. The isosensitivity curves shown in Figure 2 are assumed to be such projections.

considered so far. This modification is necessitated by one of the three defining properties of a metric: the distance between two elements of a metric space vanishes if and only if they are identical (the zero distance property, Section 3.6). In the case of subjectively unidimensional discriminations, different stimuli have a zero distance between them along the designated subjective continuum $\ell$, and, therefore, all such stimuli have to be taken to form a single element of the metric space.

We begin by assuming that, as is shown in Figure 14, the global minima of $\psi_{\mathbf{x}}(\mathbf{y})$ form an $(n-1)$-dimensional hypersurface (a line, for $n=2$ ). Then, the projection $\mathbf{P}_{\mathbf{x}}$ of this minimum-level hypersurface onto the stimulus space is also an $(n-1)$-dimensional hypersurface, and we assume that

(S1) the projections $\mathbf{P}_{\mathbf{x}}$ cover the stimulus space simply, which means that any point of the space belongs to one and only one projection.

Clearly, for any two distinct points $\mathbf{x}$ and $\mathbf{x}^{\prime}$, either $\mathbf{P}_{\mathbf{x}}=\mathbf{P}_{\mathbf{x}^{\prime}}$, or $\mathbf{P}_{\mathbf{x}} \cap \mathbf{P}_{\mathbf{x}^{\prime}}$ is empty. To be able to treat the projections as elements of a metric space, we have to assume now that

(S2) if $\mathbf{P}_{\mathbf{x}}=\mathbf{P}_{\mathbf{x}^{\prime}}$, then (at least) in a small neighborhood of this projection, $\psi_{\mathbf{x}}(\mathbf{y})=\psi_{\mathbf{x}^{\prime}}(\mathbf{y})$.

This establishes a one-to-one correspondence between the disjoint projections and distinct psychometric functions, at least in sufficiently small vicinities of the projections.

(It is possible, but not necessary for the present discussion, to employ an analogue of the relabeling argument of Sections 2.2 and 3.1 to formally ensure that $\mathbf{x} \in \mathbf{P}_{\mathbf{x}}$ or, equivalently, that $\mathbf{P}_{\mathbf{x}}$ is the set of all $\mathbf{x}^{\prime}$ such that $\mathbf{P}_{\mathbf{x}}=\mathbf{P}_{\mathbf{x}^{\prime}}$.)
Because of Property (S1), the projections of all psychometric functions can be labeled (parametrized) by a continuous variable, say, $\lambda$, assuming its values on some open interval of reals. ${ }^{9}$ The projections then can be presented as $\mathbf{P}_{\lambda}$, and the corresponding psychometric functions as $\psi_{\lambda}(\mathbf{y})$. Clearly, this parametrization induces a mapping $\Lambda$ of stimuli onto $\lambda$ values, such that $\Lambda(\mathbf{x})=\lambda$ if and only if $\mathbf{P}_{\mathbf{x}}=\mathbf{P}_{\lambda}$, and, consequently, $\psi_{\mathbf{x}}(\mathbf{y})=\psi_{\lambda}(\mathbf{y})$ (in some small vicinity of $\mathbf{P}_{\lambda}$ ).

The construction just described ensures that the parameter $\lambda$ can be viewed as a physical correlate of the subjective property $\ell$, because of which the projections $\mathbf{P}_{\lambda}$ can be viewed as isosensitivity ("equal- $\ell$ ") hypersurfaces or as hypersurfaces of $\ell$-equivalence (lines, for $n=2$ ).

Consider now some $\lambda$ and $\lambda+\Delta \lambda$, and let $\psi_{\lambda}\left(\mathbf{P}_{\lambda+\Delta \lambda}\right)$ denote the set of values of the psychometric function $\psi_{\lambda}(\mathbf{y})$ corresponding to the set of points forming $\mathbf{P}_{\lambda+\Delta \lambda}$. Obviously, $\psi_{\lambda}\left(\mathbf{P}_{\lambda}\right)$ is then the very minimum-level hypersurface of $\psi_{\lambda}(\mathbf{y})$ whose projection is $\mathbf{P}_{\lambda}$. We define the distance $\Xi(\lambda, \lambda+\Delta \lambda)$ between probability value sets $\psi_{\lambda}\left(\mathbf{P}_{\lambda}\right)$ and $\psi_{\lambda}\left(\mathbf{P}_{\lambda+\Delta \lambda}\right)$ as

$$
\Xi(\lambda, \lambda+\Delta \lambda)=\sup _{\mathbf{x} \in \mathbf{P}_{\lambda}, \mathbf{x}^{\prime} \in \mathbf{P}_{\lambda+\Delta \lambda}}\left|\psi_{\lambda}(\mathbf{x})-\psi_{\lambda}\left(\mathbf{x}^{\prime}\right)\right| .
$$

[It can be safely assumed, or derived from still weaker assumptions, that $\psi_{\lambda}\left(\mathbf{P}_{\lambda}\right)$ consists of a single value - that is, the minimum values of $\psi_{\lambda}(\mathbf{y})$ are all on the same level. This requirement is trivially satisfied, of course, if $\psi_{\lambda}(y)$ is obtained by mirror-reflection from $\gamma_{\mathbf{x}}(\mathbf{y})$, as described earlier. Denoting the single level in question by $\psi_{\lambda}$, we have then

$$
\Xi(\lambda, \lambda+\Delta \lambda)=\sup _{\mathbf{x} \in \mathbf{P}_{\lambda+\Delta \lambda}}\left|\psi_{\lambda}(\mathbf{x})-\psi_{\lambda}\right| .
$$

This simplification, both convenient and reasonable, is not, however, critical for the present discussion.]

Now we are in the position to construct the Fechnerian metric for multidimensional stimuli based on subjectively unidimensional discriminations. Note that, if in the preceding discussion one puts $n=1$, then (i) the projections $\mathbf{P}_{\lambda}$ reduce to points on a unidimensional continuum, (ii) the physical magnitudes of these points are measured by the parameter $\lambda$, (iii) $\psi_{\lambda}\left(\mathbf{P}_{\lambda+\Delta \lambda}\right)=\psi_{\lambda}(\lambda+\Delta \lambda)$ is simply the probability with which $\lambda+\Delta \lambda$ is discriminated from $\lambda$, and (iv) $\Xi(\lambda, \lambda+\Delta \lambda)$ reduces to the difference between this probability and $\psi_{\lambda}\left(\mathbf{P}_{\lambda}\right)=\psi_{\lambda}(\lambda)$. This observation makes further development rather straightforward. We assume that (for brevity's sake, the assumptions and definitions are combined below in one proposition).

(S3) there is a positive real $\mu$, the psychometric order of the stimulus space endowed with the psychometric functions $\psi_{\lambda}(\mathbf{y})$, such that the Fechner metric function

$$
F(\lambda)=\lim _{s \rightarrow 0+} \frac{\sqrt[\mu]{\Xi(\lambda, \lambda+s)}}{s}=\lim _{s \rightarrow 0+} \frac{\sqrt[\mu]{\Xi(\lambda, \lambda-s)}}{s}
$$

is finite and nonzero for all $\lambda$.

It only remains to define the Fechnerian distance between two projections $\mathbf{P}_{a}$ and $\mathbf{P}_{b}$ as 


$$
G(a, b)=\int_{a}^{b} F(\lambda) d \lambda .
$$

This integral can also be interpreted as the Fechnerian distance between any two stimuli $\mathbf{a}$ and $\mathbf{b}$ such that

$$
\Lambda(\mathbf{a})=a, \Lambda(\mathbf{b})=b .
$$

The parametrization of the projections by $\lambda$ is not, of course, unique, but it is clear, by the same argument as in Sections 2.3 and 3.5-3.6, that all (diffeomorphically related) parametrizations lead to the same Fechnerian metric.

6.2. Isosensitivity curves and Fechnerian distances. Let us return now to the situation considered in Section 1.1 and illustrated in Figure 2: two-dimensional stimuli compared with respect to a unidimensional subjective attribute $\ell$. To conform with Section 1.1, we switch from the general notation $\left(y^{1}, y^{2}\right)$ for stimulus physical coordinates to $(h, v)$ (that can be read as "horizontal--vertical"). Although no empirical work is known to us in which psychometric functions $\psi_{\lambda}(\mathbf{y})=\psi_{\lambda}(h, v)$ have actually been constructed, it seems reasonable to assume that the projections $\mathbf{P}_{\lambda}$ of these functions' minimum lines can be directly obtained by sensory-physical matching (Marks, 1974). In a typical version of this procedure, one fixes a stimulus $\mathbf{a}=\left(h_{\mathbf{a}}, v_{\mathbf{a}}\right)$ and the coordinate $h_{\mathbf{b}}$ of a stimulus $\mathbf{b}=\left(h_{\mathbf{b}}, v_{\mathbf{b}}\right)$, and one adjusts its other coordinate, $v_{\mathbf{b}}$, so that $\mathbf{a}$ and $\mathbf{b}$ appear $\ell$-equivalent. Repeating this procedure for different values of $h_{\mathrm{b}}$, one gets a curve of stimuli, all of which are $\ell$-equivalent to a. A family of "equal- $\ell$ " curves, like the one shown in Figure 2, is obtained by varying the coordinate $v_{\mathrm{a}}$, which thereby plays the role of the parameter $\lambda$. This being a popular psychophysical technique, a great variety of equal- $\ell$, or isosensitivy, curve families have been reported in the literature (see, e.g., Marks, 1974). Among them of particular interest to us are equal-loudness (or isosonic) curves for pure tones, where $h$ is frequency and $v$ is intensity (Fletcher \& Munson, 1933), because these curves, taken in conjunction with JNDs in sound intensity measured at different frequencies (Riesz, 1933), were used in the historically first attempt to empirically test Fechner's postulate (in its traditional understanding; see Section 2.1).

As mentioned in Section 1.1, Riesz's (1933) measurements show that the numbers of the intensity JNDs between two isosonic curves are different at different frequencies. On the basis of Riesz's measurements, Stevens (1936) derives an empirical formula that allows one to compute, for several frequencies, the number of justnoticeable intensity increments between zero loudness (i.e., the absolute threshold of intensity) and any given loudness level (measured in sones). According to this formula, for any given loudness level, this number of justnoticeable intensity increments for a $1000-\mathrm{Hz}$ tone is roughly 2.3 times greater than for a $200-\mathrm{Hz}$ tone and roughly 1.9 times smaller than for a $4000-\mathrm{Hz}$ tone. Most psychophysicists consider this an unequivocal refutation of Fechner's postulate. Note that the inconsistency found here is between two comparable judgment schemes: the "greater than-less than" judgments required for measuring the intensity JNDs and the same-different judgments required for constructing the isosonic curves. This comparability makes the criticism in question considerably more convincing than any discrepancy found between Fechnerian distances and those computed from, say, direct magnitude estimates.

The logic of this criticism, however, is flawed. To keep the discussion interesting, let us assume that the finite just-noticeable increments in the value of $v$ (refer to Figure 2) are roughly proportional to the corresponding unidimensional Fechner metric functions (as discussed in Section 2.1, in the context of relating the infinitesimal version of Fechner's postulate to its traditional interpretation). If this assumption is incorrect, the discussion ends here, because then the number of JNDs cannot be used as an estimate of the unidimensional Fechnerian distances. Having made this assumption, the empirical fact just described indicates that the Fechnerian distance between $\mathbf{a}_{1}$ and $\mathbf{a}_{2}$ on the unidimensional continuum $h=h_{1}$ is numerically different from the Fechnerian distance between $\mathbf{b}_{\mathbf{1}}$ and $\mathbf{b}_{\mathbf{2}}$ on the unidimensional continuum $h=$ $h_{2}$. Recall, however, that the precise meaning of the Fechnerian distances here must be derived from unidimensional psychometric functions $\psi_{v}(v+\Delta v)$ on these two continua. These unidimensional distances are logically unrelated to the Fechnerian distances derived from twodimensional psychometric functions $\psi_{\lambda}(h, v)$, like the one shown in Figure 14. According to the theory presented in the previous section, the Fechnerian distance between the loudness levels of $\mathbf{a}_{1}$ and $\mathbf{a}_{\mathbf{2}}$ in the two-dimensional space $(h, v)$ must be computed as the Fechnerian distance between the entire isosonic curves containing the two points: This guarantees that the Fechnerian distance is the same for all four pairs $\left(\mathbf{a}_{1}, \mathbf{a}_{2}\right),\left(\mathbf{b}_{1}, \mathbf{b}_{2}\right),\left(\mathbf{a}_{1}, \mathbf{b}_{2}\right),\left(\mathbf{b}_{1}, \mathbf{a}_{2}\right)$.

If this analysis appears insufficiently intuitive, the following informal argument may be helpful. Assume that, referring to Figures 2 and 15, the Fechnerian distances were computed between individual points (say, $\mathbf{a}_{1}$ and $\mathbf{a}_{2}$ ) rather than the entire equal- $\ell$ curves containing them. Then, according to the general theory (Section 3 ), the distance between $\mathbf{a}_{1}$ and $\mathbf{a}_{2}$ had to be computed as the infimum of the length of all paths connecting $\mathbf{a}_{1}$ with $\mathbf{a}_{\mathbf{2}}$ in the twodimensional space. It is easy to see that this infimum length must be precisely the same for $\left(\mathbf{a}_{1}, \mathbf{a}_{2}\right)$ and $\left(\mathbf{b}_{1}, \mathbf{b}_{2}\right)$. Indeed, given any path $\mathbf{a}_{1} \rightarrow \mathbf{a}_{2}$ connecting these two points, one can connect $\mathbf{b}_{1}$ with $\mathbf{b}_{2}$ by the path

$$
b_{1} \rightarrow a_{1} \rightarrow a_{2} \rightarrow b_{2},
$$

in which the first and the third limbs $\left(\mathbf{b}_{1} \rightarrow \mathbf{a}_{1}\right.$ and $\mathbf{a}_{2} \rightarrow$ $b_{2}$ ) are taken along the equal- $\ell$ curves and have, therefore, the Fechnerian length of zero. Since this path has the same length as $\mathbf{a}_{1} \rightarrow \mathbf{a}_{\mathbf{2}}$, the infimum length for all pos- 

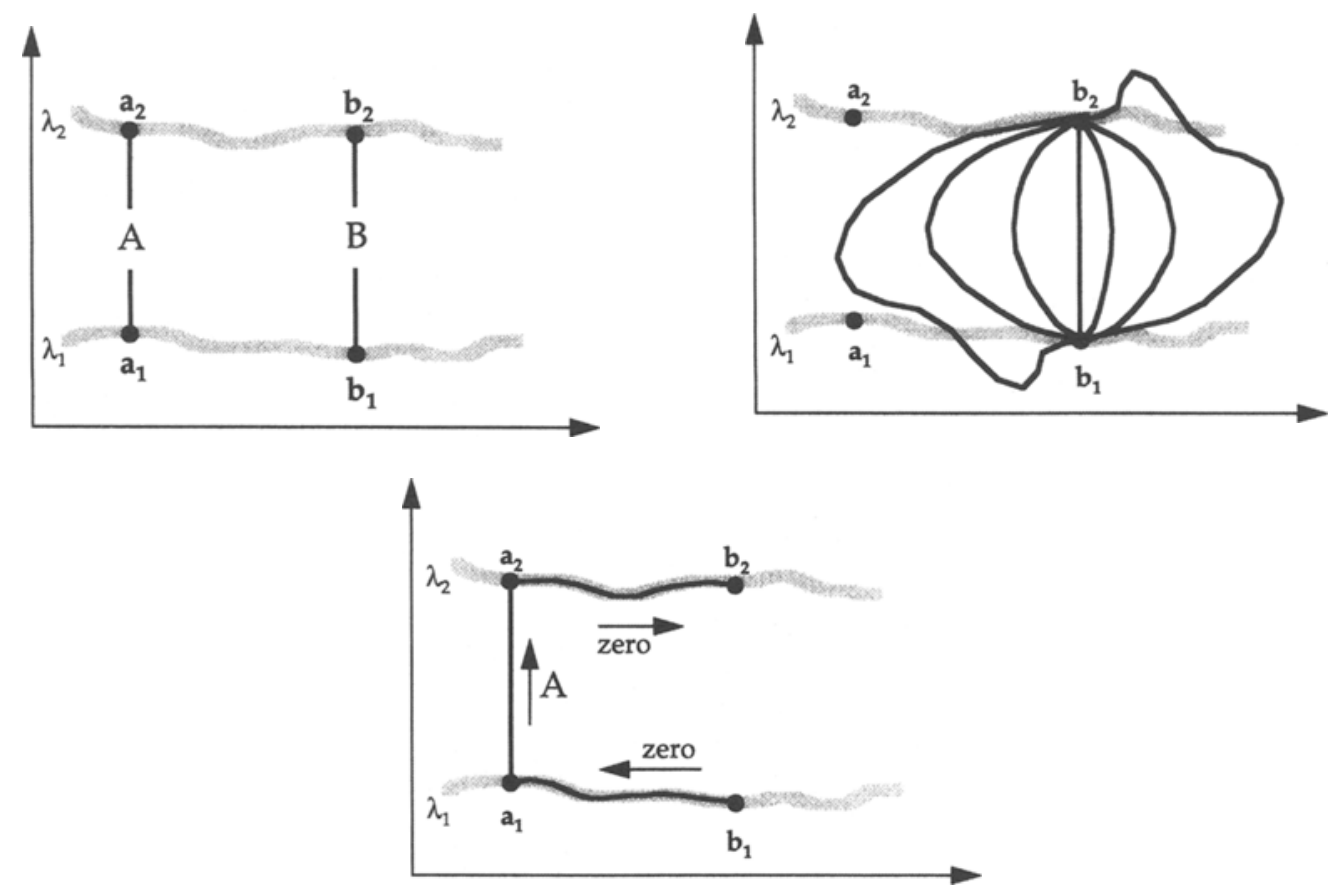

\begin{abstract}
Figure 15. Illustration for the informal argument of Section 6.2. The isosensitivity curves are taken from Figure 2; $A$ and $B$ are subjective lengths of the vertical segments $a_{1} \rightarrow a_{2}$ and $b_{1} \rightarrow b_{2}$, respectively. If $B>A$, then $B$ cannot be the Fechnerian distance between $b_{1}$ and $b_{2}$, because, among all possible paths connecting $b_{1}$ and $b_{2}$ (right panel), there is at least one (bottom panel) whose length is less than $B$. The argument is informal because one cannot speak of distances if they vanish between nonidentical points.
\end{abstract}

sible paths $\mathbf{b}_{1} \rightarrow \mathbf{b}_{2}$ cannot exceed the infimum for $\mathbf{a}_{1} \rightarrow$ $\mathbf{a}_{2}$. Then, by symmetry, the two infima must coincide.

\section{Conclusion}

7.1. Comeasurability in the small. The central notion in our theory of Fechnerian scaling is that of the comeasurability in the small, of the distance $s$ between stimuli $\mathbf{x}$ and $\mathbf{x}+\mathbf{u} s$ with the (generally transformed) distance between the corresponding psychometric values, $\psi_{\mathbf{x}}(\mathbf{x})$ and $\psi_{\mathbf{x}}(\mathbf{x}+\mathbf{u} s)$ :

$$
\Phi\left[\psi_{\mathbf{x}}(\mathbf{x}+\mathbf{u} s)-\psi_{\mathbf{x}}(\mathbf{x})\right]=O(s), s \rightarrow 0+.
$$

As explained in Section 3.2, such a transformation can always be found for a given stimulus $\mathbf{x}$ and a given (nonzero) direction $\mathbf{u}$. It is not logically necessary, however, that this transformation be the same for all stimuli and all directions. The assumption that this is de facto the case is critical for the theory, because it enables one to say that the subjective values of the infinitesimally small distances in a stimulus space can be measured by the differentials

$$
\Phi\left[\psi_{\mathbf{x}}(\mathbf{x}+\mathbf{u} s)-\psi_{\mathbf{x}}(\mathbf{x})\right], s \rightarrow 0+.
$$

Psychometric functions are based on the counting of cases when $\mathbf{x}+\mathbf{u} s$ is judged to be different from $\mathbf{x}$, because of which the differentials of psychometric values are completely independent of the physical characteristics of $\mathbf{x}$ and the measurement procedures by which the coordinates of $\mathbf{x}$ are computed. Fechnerian scaling can be said to be providing a universal yardstick by which to measure subjective distances among stimuli without regard to how they have been measured physically. This yardstick is utilized by, first, introducing the FechnerFinsler function

$$
F(\mathbf{x}, \mathbf{u})=\lim _{s \rightarrow 0+} \frac{\Phi\left[\psi_{\mathbf{x}}(\mathbf{x}+\mathbf{u} s)-\psi_{\mathbf{x}}(\mathbf{x})\right]}{s},
$$

then deriving a subjective (or psychometric) distance between any two points in the stimulus space,

$$
G(\mathbf{a}, \mathbf{b})=\inf _{\mathbf{a}(t)_{\mathbf{b}}}\left\{\int_{a}^{b} F(\mathbf{x}(t), \dot{\mathbf{x}}(t)) d t\right\},
$$

and, finally, proving that $G(\mathbf{a}, \mathbf{b})$ is a valid metric in the stimulus space, provided $F(\mathbf{x}, \mathbf{u})$ is endowed with suitable properties (such as smoothness and symmetry). See Section 3 for details.

It is easy to understand why it is critical that the transformation $\Phi$ be the same throughout all points and directions in a stimulus space. The logic of the comeasurability statement allows for the transformation $\Phi$ to be multiplied by an arbitrary positive constant:

$$
\begin{aligned}
& \Phi\left[\psi_{\mathbf{x}}(\mathbf{x}+\mathbf{u} s)-\psi_{\mathbf{x}}(\mathbf{x})\right]=O(s), s \rightarrow 0^{+} \\
& +\Leftrightarrow k \Phi\left[\psi_{\mathbf{x}}(\mathbf{x}+\mathbf{u} s)-\psi_{\mathbf{x}}(\mathbf{x})\right]=O(s), s \rightarrow 0+.
\end{aligned}
$$

If a single transformation that applies to all stimuli and directions can be chosen, then one can set the coefficient $k$ equal to one and the same constant (say, unity) everywhere. Then the Fechner-Finsler function is defined uniquely, 
and the same is true for the corresponding Fechnerian metric:

$$
\inf _{\mathbf{a}\left(t_{\mathbf{b}}\right.}\left\{\int_{a}^{b} k F(\mathbf{x}(t), \dot{\mathbf{x}}(t)) d t\right\}=k G(\mathbf{a}, \mathbf{b}) .
$$

If, however, there is no universally applicable single transformation $\Phi$, then the notion of a common scaling factor becomes meaningless, and one is free to define different similarity transformations for different stimuli and directions. The Fechner-Finsler function then, rather than being multiplied by an arbitrary positive coefficient, can now be multiplied by an arbitrary positive function,

$$
k(\mathbf{x}, \mathbf{u}) F(\mathbf{x}, \mathbf{u}),
$$

which is equivalent to saying that the Fechner-Finsler function itself is arbitrary.

We have assumed throughout this paper that the transformation of the psychometric differentials that makes them comeasurable with the transition from $\mathbf{x}$ to $\mathbf{x}+\mathbf{u s}$ is a power function,

$$
\Phi\left[\psi_{\mathbf{x}}(\mathbf{x}+\mathbf{u} s)-\psi_{\mathbf{x}}(\mathbf{x})\right]=\sqrt[\mu]{\psi_{\mathbf{x}}(\mathbf{x}+\mathbf{u} s)-\psi_{\mathbf{x}}(\mathbf{x})} .
$$

As explained in Section 2.2, this assumption is very unrestrictive, which can be easily appreciated on observing that it is satisfied whenever, at $s=0, \psi_{\mathbf{x}}(\mathbf{x}+\mathbf{u} s)$ has a nonzero finite derivative of some order with respect to $s$ (in which case the lowest order for which this is true is the psychometric order $\mu$ ). Power transformations, however, are not applicable universally. As an example, if

$$
\psi_{\mathbf{x}}(\mathbf{x}+\mathbf{u} s)-\psi_{\mathbf{x}}(\mathbf{x})=O\left(s^{1 / s}\right), s \rightarrow 0+,
$$

then any power transformation of the psychometric differential has a higher degree of infinitesimality than $s$.

A careful inspection shows that no assumptions or derivations constituting our theory are critically based on the choice of power transformations and that the theory can be rewritten with no further modifications if the power transformations are replaced everywhere with an arbitrary transformation $\Phi$. The only and minor exception is

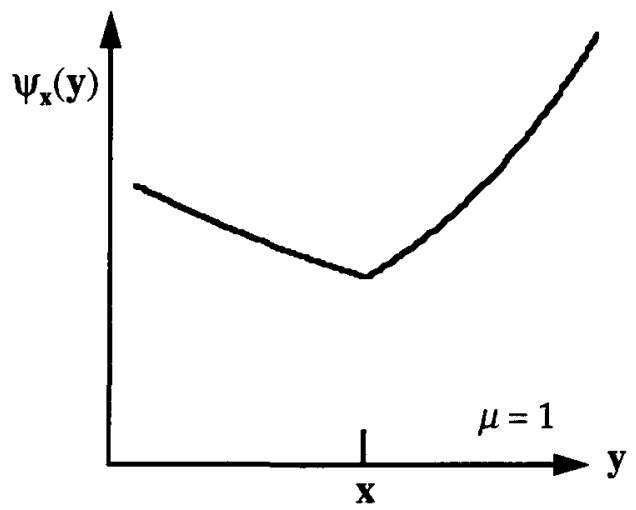

the criterion of Fechner-equivalence introduced in Section 3.7 and used in Section 5. The initial formulation,

$$
\begin{aligned}
F(\mathbf{x}, \mathbf{u}) & =k \bar{F}(\mathbf{x}, \mathbf{u}) \Leftrightarrow \frac{\sqrt[\mu]{\bar{\psi}_{\mathbf{x}}(\mathbf{x}+\mathbf{u} s)-\bar{\psi}_{\mathbf{x}}(\mathbf{x})}}{\sqrt[\mu]{\psi_{\mathbf{x}}(\mathbf{x}+\mathbf{u} s)-\psi_{\mathbf{x}}(\mathbf{x})}} \\
& =k+o\{\mathbf{l}\}, s>0,
\end{aligned}
$$

can be trivially replaced with

$$
\begin{aligned}
F(\mathbf{x}, \mathbf{u}) & =k \bar{F}(\mathbf{x}, \mathbf{u}) \Leftrightarrow \frac{\bar{\Phi}\left[\psi_{\mathbf{x}}(\mathbf{x}+\mathbf{u} s)-\psi_{\mathbf{x}}(\mathbf{x})\right]}{\Phi\left[\psi_{\mathbf{x}}(\mathbf{x}+\mathbf{u} s)-\psi_{\mathbf{x}}(\mathbf{x})\right]} \\
& =k+o\{1\}, s>0,
\end{aligned}
$$

but the subsequent reformulations derived in Sections 3.7 and 5 make use of power functions' properties. One does not, however, lose any substantive points by dispensing with these reformulations.

7.2. Fechnerian metric without symmetry. Another constraint that has been assumed to hold throughout the paper but is not logically necessary for the construction of the Fechnerian metric is the symmetry assumption FF2 of Section 3.4. The following is a sketch of the theory that does not assume the symmetry. Refer to Figure 16.

The main difference caused by dropping the symmetry assumption is that Property 33 , saying that the Finslerian length of a path is independent of the sense of traversing it, holds no longer. One can remedy this situation by redefining the Finslerian length $\left.\mathfrak{I}_{\mathbf{a}} \mathbf{x}(t)_{\mathbf{b}}\right]$ while preserving the definition of the Fechnerian distance as the infimum of these values. The simplest way is, of course, to put

$$
\left.\mathfrak{I}^{*}{ }_{\mathbf{a}_{\mathbf{a}}} \mathbf{x}(t)_{\mathbf{b}}\right]=\max \left\{\mathfrak{I}\left[_{\mathbf{a}} \mathbf{x}(t)_{\mathbf{b}}\right], \mathfrak{I}\left[{ }_{\mathbf{b}} \mathbf{x}(t)_{\mathbf{a}}\right]\right\} .
$$

Obviously,

$$
\left.\mathfrak{I} *{ }_{\mathbf{a}} \mathbf{x}(t)_{\mathbf{b}}\right]=\mathfrak{I}^{*}\left[\mathbf{b} \mathbf{x}(t)_{\mathbf{a}}\right],
$$

and if the symmetry constraint is satisfied, $\mathfrak{I}^{*}\left[_{\mathbf{a}} \mathbf{x}(t)_{\mathbf{b}}\right]$ reduces to $\mathfrak{I}\left[\mathbf{a} \mathbf{x}(t)_{\mathbf{b}}\right]$.

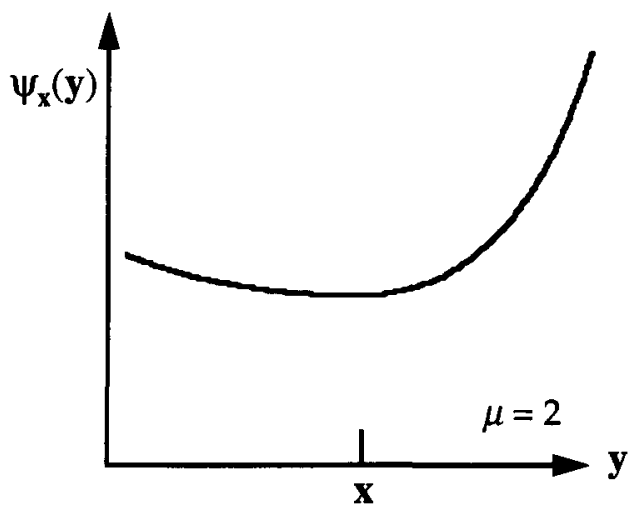

Figure 16. Asymmetric psychometric functions with different values of $\mu$ in a small vicinity of their global minima (vertical cross sections through the minima). 


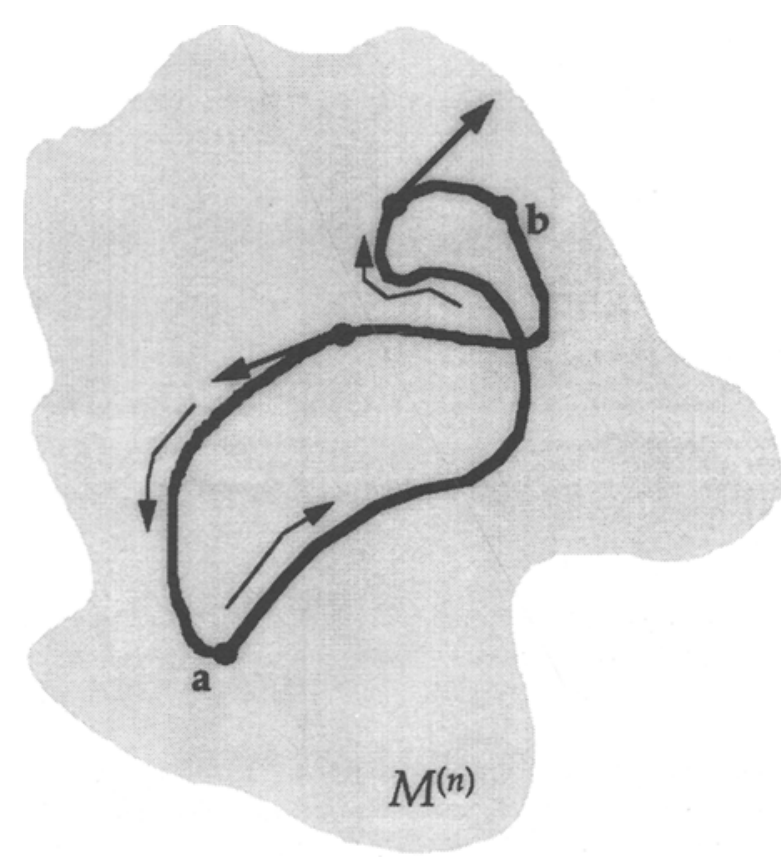

Figure 17. A closed path containing points a and $b$ and traversed in a certain direction taken to be positive. Its psychometric length is computed in the same way as in Figure 13.

Another way is to define $\mathfrak{I}^{*}\left[{ }_{\mathbf{a}} \mathbf{x}(t)_{\mathbf{b}}\right]$ as the length of $\mathbf{a}$ closed path ${ }_{\mathrm{a}} \mathbf{x}(t)_{\mathrm{b}}$ containing the two points and traversed in a certain direction taken to be positive (see Figure 17):

$$
\mathfrak{I} *\left[\mathbf{a}(t)_{\mathbf{b}}\right]=\oint F(\mathbf{z}(t), \dot{\mathbf{z}}(t)) d t .
$$

Again, this approach guarantees

$$
\mathfrak{S} *\left[_{\mathbf{a}} \mathbf{z}(t)_{\mathbf{b}}\right]=\mathfrak{S}^{*}\left[\mathbf{b} \mathbf{z}(t)_{\mathbf{a}}\right]
$$

In the symmetrical case, this quantity reduces to $2 \mathfrak{I}\left[{ }_{\mathbf{a}} \mathbf{x}(t)_{\mathbf{b}}\right.$ ] (because of which it may be desirable to define the $\mathfrak{I}^{*}\left[{ }_{\mathbf{a}} \mathbf{z}(t)_{\mathbf{b}}\right]$ as the half-length of the closed path containing the two points).

7.3. Why not just Riemannian? The Fechnerian metric constructed in our theory is mathematically Finslerian. By definition (Section 3.4), it becomes Riemannian if and only if the metric tensor

$$
g_{i j}(\mathbf{x}, \mathbf{u})=\frac{1}{2} \frac{\partial^{2} F(\mathbf{x}, \mathbf{u})^{2}}{\partial u^{i} \partial u^{j}}, i, j=1, \ldots, n
$$

at any point $\mathbf{x}$ of a stimulus space does not depend on the direction $\mathbf{u}$ :

$$
g_{i j}(\mathbf{x}, \mathbf{u}) \equiv g_{i j}(\mathbf{x}), i, j=1, \ldots, n .
$$

Since the Riemannian geometry is by far better known than the Finslerian one, at least in psychophysical applications (see Section 4.2), the question arises: Why do we not simply posit that the metric tensor $g_{i j}(\mathbf{x}, \mathbf{u})$ is directionindependent and thereby confine our analysis to the Riemannian metric only? After all, one might say, would not such an assumption be as innocuous as the smoothness conditions that we impose on the shape of the psychometric functions $\psi_{\mathbf{x}}(\mathbf{y})$ and on the metric function $F(\mathbf{x}, \mathbf{u})$ ?

The answer to this question does not lie in our wish to be as general as possible: More general structures than the Finslerian geometry are readily available. The essential
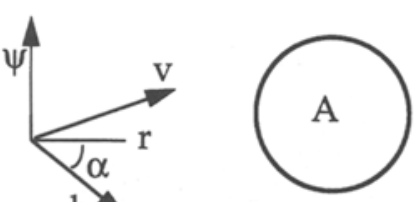

$\mathrm{h}$
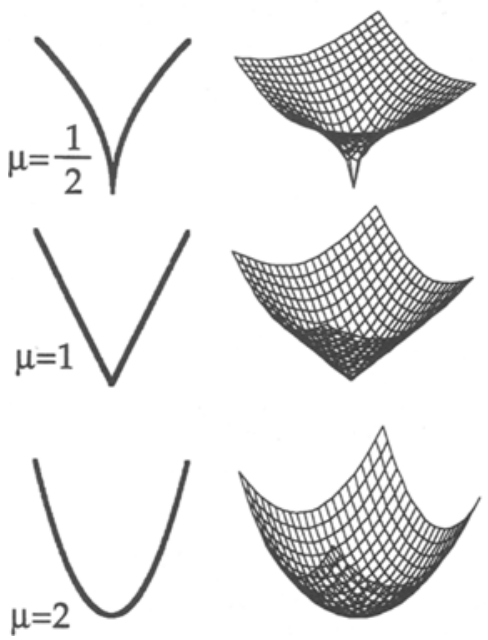
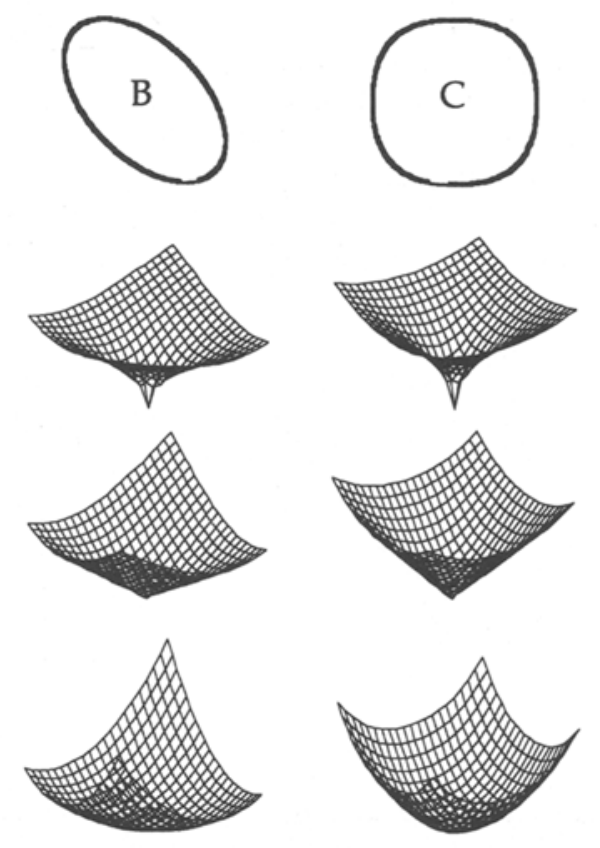

Figure 18. Psychometric functions in a small vicinity of their minima with different shapes of the vertical cross sections through their minima (left margin) and horizontal cross sections near their minima (upper margin). See Section 7.3 for details. 
point is that, unlike Helmholtz (1891) and Schrödinger (1920), we do not depart from the assumption that the subjective metric of a stimulus space has any particular geometric structure, and geometric concepts, such as metric tensors and geodesics, are not theoretical primitives of our analysis. Rather we depart from the observable psychometric functions and from the idea of measuring infinitesimal changes in stimuli by the (transformed) psychometric differentials. We introduce a function $F(\mathbf{x}, \mathbf{u})$ that allows us to do this measuring, and it turns out to be the metric function of the Finslerian geometry. To make this function theoretically manageable, we impose on it certain regularity conditions, such as the assumption that $F(\mathbf{x}, \mathbf{u})$ is differentiable sufficient number of times with respect to its arguments. There is indeed no obvious danger in making such smoothness constraints arbitrarily strong: It would have been relatively innocuous, for instance, to assume that $F(\mathbf{x}, \mathbf{u})$ is infinitely differentiable in all arguments.

The situation, however, is very different if one postulates the direction-independence of the metric tensor $g_{i j}(\mathbf{x}, \mathbf{u})$. This postulate imposes constraints on the shape of the psychometric functions that cannot be a priori justified by any substantive or mathematical arguments beyond one's sheer desire to ensure that the resulting Fechnerian metric be Riemannian. Figure 18 illustrates this point. The two-dimensional psychometric functions $\psi_{\mathbf{x}}(\mathbf{y})$ shown are grouped into columns according to the shape of the horizontal cross sections made near their minima and into rows according to the psychometric order $\mu$ of the stimulus space (we return here to the power function specialization of the transformation $\Phi$ ). The psychometric order determines the shape of the vertical cross sections made through the minima of the psychometric functions. Placing, for simplicity, the reference stimuli at the origin,

$$
\mathbf{x}=(0,0), \psi_{0,0}(0,0)=0,
$$

and renaming $\mathbf{y}=\left(y^{1}, y^{2}\right)$ into $(h, v)$, the analytic expressions for these psychometric functions are

$$
\begin{aligned}
& \psi_{(0,0)}^{(A)}(h, v)=\left(h^{2}+v^{2}\right)^{\mu / 2} \\
& \psi_{(0,0)}^{(B)}(h, v)=\left(h^{2}+v^{2}+h v\right)^{\mu / 2} \\
& \psi_{(0,0)}^{(C)}(h, v)=\left(h^{4}+v^{4}+h^{2} v^{2}\right)^{\mu / 4} \\
& \psi_{(0,0)}^{(D)}(h, v)=\left[\left(h^{2}+v^{2}+h v\right)\left(h^{2}+v^{2}\right)\right]^{\mu / 4},
\end{aligned}
$$

where the superscript at $\psi$ refers to the column and $\mu$ refers to the row (note that all the superscripts at $h$ and $v$ are exponents). The Fechner-Finsler metric functions

$$
F(\mathbf{x}, \mathbf{u})=F\left[(0,0),\left(u^{1}, u^{2}\right)\right]
$$

can be obtained from these formulas by simply replacing $(h, v)$ with $\left(u^{1}, u^{2}\right)$ and putting $\mu=1$. They do not, of course, depend on the psychometric order and are shared by all psychometric functions belonging to the same column.

It is easy to verify that the metric tensor $g_{i j}(\mathbf{x}, \mathbf{u})$ computed from these metric functions is direction-independent for the columns A and B, but not for C and D. This implies that the Fechnerian metric is Riemannian for a stimulus space whose psychometric functions are, say, of the shape

$$
\sqrt[4]{h^{2}+v^{2}+h v}
$$

(column B, $\mu=1 / 2$ ), whereas it is not Riemannian if the shape is, say,

$$
\sqrt[4]{\left(h^{2}+v^{2}+h v\right)\left(h^{2}+v^{2}\right)}
$$

(column D, $\mu=2$ ). In polar coordinates, if one expresses the value of the psychometric function $\psi_{(0,0)}(h, v)$ through the length $r$ and angle $\alpha$ of the vector $(h, v)$, these two shapes are described by, respectively,

$$
\sqrt{r} \sqrt[4]{1+\frac{1}{2} \sin 2 \alpha} \text { and } r^{2} \sqrt{1+\frac{1}{2} \sin 2 \alpha} .
$$

We can think of no reason for declaring a priori that only the former but not the latter may exist empirically. Note that all the psychometric functions and the corresponding Fechner-Finsler functions in our example are equally "well-behaved": They are all infinitely differentiable at nonzero values of their arguments, while the degree of smoothness at the minima of the psychometric functions is determined solely by the psychometric order $\mu$ and has nothing to do with the difference between the column pairs $\mathrm{A}-\mathrm{B}$ and $\mathrm{C}-\mathrm{D}$.

7.4. Discrimination schemes, estimated distances, and Fechnerian scaling. A careful examination of the assumptions underlying Fechnerian scaling (Sections 3 and 6) shows that all of them refer to observable properties of psychometric functions and can, at least in principle, be shown to be false. ${ }^{10}$ Most of them, however, are properties of infinitesimally small areas of psychometric functions, and, as stated in Section 1.3, their corroboration may require very large experiments and additional technical assumptions. Moreover, it has been shown that some of the underlying assumptions of the theory (power transformations, symmetry) can be relaxed and generalized with no serious consequences. Therefore, in Section 1.3, we define the main intended contribution of our theory in terms of conceptual clarity rather than falsifiable predictions. We believe that a century and a half of the continuing controversy surrounding Fechnerian scaling, with a good deal of repetition and flawed reasoning involved, testifies to the importance of striving to discuss the issues in clear and rigorous terms. This is where lies the principal value of such landmarks in the history of the controversy as Falmagne $(1971,1985)$, Luce and Edwards (1958), and Pfanzagl (1962).

We think that the ultimate usefulness of our approach to Fechnerian scaling hinges not so much on the empiri- 
cal corroboration of its underlying assumptions (which is, of course, a necessary condition) as on the scope and diversity of the experimental paradigms whose outcomes can be related to Fechnerian distances in a lawful fashion. It has been repeatedly stated in this paper, for instance, that different discrimination judgments may lead to different Fechnerian metrics on one and the same stimulus space. This does not mean, however, that they have to be unrelated, and empirically testable models may be constructed as predicting particular relationships among (perhaps the identity of) Fechnerian metrics derived from different classes of psychometric functions. Lawful relationships may also be expected to exist between Fechnerian metrics derived from psychometric functions and outcomes of other discriminability-related measurements (such as the methods of limits and adjustments). Such relationships would have enormously simplified the computation of Fechnerian distances and, among other things, would have made higher dimensional stimulus spaces accessible to practical experimentation.

It will also be important to find out whether Fechnerian distances can be shown to underlie subjective estimates of large dissimilarities among stimuli (as those obtained by multidimensional scaling techniques). Could, for example, such a subjective dissimilarity measure between $\mathbf{a}$ and $\mathbf{b}$ be a function of $G(\mathbf{a}, \mathbf{b})$ or of $G(\mathbf{a}, \mathbf{b})$ taken in conjunction with Fechnerian distances $G(\mathbf{a}, \mathbf{o})$ and $G(\mathbf{b}, \mathbf{0})$ from a fixed point 0 in the stimulus space? Such relationships too may be targeted by empirically falsifiable models, very much in the spirit of the admittedly naive but ingenious derivation of Stevens's power function from the equality of two Fechner's logarithmic functions (see Baird, 1997, pp. 92-95; Ekman, 1964; Laming, 1997, ch. 8; Luce \& Galanter, 1963). A sufficiently rich network of empirically testable relationships among Fecherian metrics derived from a variety of discrimination schemes and large dissimilarity estimates may conceivably lead to a rigorous theory of true (without quotation marks) subjective distances, thereby achieving the ultimate aim of Fechner's seminal endeavor.

\section{REFERENCES}

Baird, J. C. (1997). Sensation and judgment: Complementarity theory of psychophysics. Mahwah, NJ: Erlbaum.

CARATHÉODORY, C. (1982). Calculus of variations and partial differential equations of the first order. New York: Chelsea.

Creelman, C. D. (1967). Empirical detectability scales without the jnd. Perceptual \& Motor Skills, 24, 1079-1084.

DzhaFARov, E. N. (1993). Grice-representability of response time distribution families. Psychometrika, 58, 281-314.

Ekman, G. (1964). Is the power law a special case of Fechner's law? Perceptual \& Motor Skills, 19, 730.

ElSAss, A. (1886). Über die Psychophysik. Physikalische und erkenntnistheoretische Betrachtungen [On psychphysics. Physical and epistemological studies]. Marburg: Elwert.

FALMAGNE, J. C. (1971). The generalized Fechner problem and discrimination. Journal of Mathematical Psychology, 8, 22-43.

FALMAGNE, J. C. (1985). Elements of psychophysical theory. Oxford: Oxford University Press.

FECHNER, G. T. (1851). Zend-Avesta; oder über die Dinge des Himmels und des Jenseits [Zend-Avesta, or on matters of heaven and the be- yond]. Leipzig: Voss. (Vol. 2, pp. 373-386; translated and edited by E. Scheerer, Psychological Research, 1987, 49, 203-207)

FECHNER, G. T. (1860). Elemente der Psychophysik [Elements of psychophysics]. Leipzig: Breitkopf \& Härtel.

FeCHNER, G. T. (1877). In Sachen der Psychophysik [In the matter of psychophysics]. Leipzig: Breitkopf \& Härtel.

FECHNER, G. T. (1887). Über die psychischen Massprinzipien und das Webersche Gesetz [On the principles of mental measurement and Weber's Law]. Philosophische Studien, 4, 161-230. (Excerpts from pp. 178-198; translated and edited by E. Scheerer, Psychological Research, 1987, 49, 213-219)

FLETCHER, H., \& MUNSON, W. A. (1933). Loudness, its definition, measurement and calculation. Journal of the Acoustical Society of America, 5, 82-108.

GeLfand, I. M., \& Fomin, S. V. (1963). Calculus of variations. Englewoods Cliffs, NJ: Prentice-Hall

GUILFORD, J. P. (1954). Psychometric methods. New York: McGraw-Hill. HeLMHOLTZ, H. vON (1891). Versuch einer erweiterten Anwendung des Fechnerschen Gesetzes im Farbensystem [An attempt at a generalized application of Fechner's Law to the color system]. Zeitschrift für die Psychologie und die Physiologie der Sinnesorgane, 2, 1-30.

INDOW, T. (1993). Indiscriminable regions, color differences, and principal hue vectors in Munsell space. Die Farbe, 39, 15-23.

InDow, T., \& MorRISON, M. L. (1991). Construction of discrimination ellipsoids for surface colors by the methods of constant stimuli. Color Research \& Applications, 16, 42-56.

Indow, T., Robertson, A. R., von Grunau, M., \& Fielder, G. H. (1992). Discrimination ellipsoids of aperture and simulated surface colors by matching and paired comparison. Color Research \& Applications, 17, 6-23.

KRANTZ, D. (1971). Integration of just-noticeable differences. Journal of Mathematical Psychology, 8, 591-599.

KREYSZIG, E. (1968). Introduction to differential geometry and Riemannian geometry. Toronto: University of Toronto Press.

KrUEger, L. E. (1989). Reconciling Fechner and Stevens: Toward a unified psychophysical law. Behavioral \& Brain Sciences, 12, 251-320.

LAMING, D. (1997). The measurement of sensation. Oxford: Oxford University Press.

LEVIN, D. (in press). A differential geometric description of the relationships among perceptions. Journal of Mathematical Psychology.

LEVINE, M. (1970). Transformations that render curves parallel. Journal of Mathematical Psychology, 7, 410-443.

Link, S. W. (1992). The wave theory of difference and similarity. Hillsdale, NJ: Erlbaum.

LoCKHEAD, G. R. (1992). Psychophysical scaling: Judgments of attributes or objects? Behavioral \& Brain Sciences, 15, 543-601.

LUCE, R. D., \& EDWARDS, W. (1958). The derivation of subjective scales from just noticeable differences. Psychological Review, 65, 222-237.

Luce, R. D., \& Galanter, E. (1963). Discrimination. In R. D. Luce, R. R. Bush, \& E. Galanter (Eds.), Handbook of mathematical psychology (Vol. 1, pp. 191-244). New York: Wiley.

MARKS, L. E. (1974). Sensory processes: The new psychophysics. New York: Academic Press.

Murray, D. J. (1992). A perspective for viewing the history of psychophysics. Behavioral \& Brain Sciences, 16, 115-137.

Nachmias, J., \& Steinman, R. M. (1965). Brightness and discriminability of light flashes. Vision Research, 5, 545-557.

PFANZAGL, J. (1962). Über die stochastische Fundierung des psychophysischen Gesetzes [On stochastic foundations of the psychophysical law]. Biometrische Zeitschrift, 4, 1-14.

RiEsz, R. R. (1933). The relationship between loudness and the minimum perceptible increment of intensity. Journal of the Acoustical Society of America, 4, 211-216.

RoBERTSON, A. R. (1978). CIE guidelines for coordinated research on colour-difference evaluation. Color Research \& Applications, 3, 149-151.

Rund, H. (1959). The differential geometry of Finsler spaces. Berlin: Springer-Verlag.

SCHEERER, E. (1987). The unknown Fechner. Psychological Research, 49, 197-202. 
SCHRÖDINGER, E. VON (1920). Farbenmetrik [Color metrics]. Zeitschrift für Physik, 12, 459-466.

STEVENS, S. S. (1936). A scale for the measurement of a psychological magnitude: Loudness. Psychological Review, 43, 405-416.

StEvens, S. S. (1975). Psychophysics: Introduction to its perceptual, neural, and social prospects. New York: Wiley.

WITT, K., \& DöRING, G. (1983). Parametric variations in a threshold color-difference ellipsoid for green painted samples. Color Research \& Applications, 8, 153-163.

\section{NOTES}

1. The reader is reminded that if $\alpha(x) \rightarrow 0, \beta(x) \rightarrow 0$ (as $x \rightarrow x_{0}$ ), then one writes $\beta=o(\alpha)$ to designate that $\beta(x) / \alpha(x) \rightarrow 0$, or $\alpha(x) / \beta(x) \rightarrow$ $\pm \infty$ (as $x \rightarrow x_{0}$ ), and one says that $\beta$ has a higher order of infinitesimality than $\alpha$.

2 . In Section 3, this concept is further generalized into that of the Fechner-Finsler metric function.

3. We remind the reader that if $\alpha(x) \rightarrow 0, \beta(x) \rightarrow 0$ (as $x \rightarrow x_{0}$ ), then one writes $\beta=O(\alpha)$ to designate that $\beta(x) / \alpha(x)$ tends to a finite nonzero value (as $x \rightarrow x_{0}$ ), and one says then that the two infinitesimals are of the same order of infinitesimality. If the limit of $\beta(x) / \alpha(x)$ is a positive number, then we call the two infinitesimals comeasurable (at $\left.x=x_{0}\right)$. Obviously, for nonnegative infinitesimals, $\beta=O(\alpha)$ implies comeasurability.

4. If we did not specialize the transformation $\Phi$, the assumption would have been that $\Phi$, whatever it might be, is one and the same for all psychometric functions.

5. After P. Finsler, who proposed a generalization of Riemannian geometry in 1918 (see Rund, 1959). Obviously, the term FechnerFinsler does not imply a collaboration or parallel discovery. Rather it reflects the fact that we deal with Finsler's metric functions of a special origin, computed from psychometric functions, and with a special purpose, to compute Fechnerian distances. Unlike in abstract geometry, the metric function in our theory is not a conceptual primitive, and its most fundamental properties (the Euler homogeneity, positivity, and invariance, considered later) are derived from the properties of the psychometric functions, rather than postulated.

6 . Recall that a function is called $C^{r}$ if it has continuous partial derivatives of the $r$ th order.

7. Diffeomorphic transformation means a one-to-one transformation with a nonvanishing Jacobian and such that both the transformation and its inverse are (at least) $C^{1}$.

8. This does not mean that, without the assumption of positive regularity, one cannot compute $G(\mathbf{a}, \mathbf{b})$ as the infimum of a series of path lengths: Under certain conditions, one can in fact effectively construct such a sequence and approximate its limit. See, as one possible approach, the discussion of the "direct methods of variational calculus" in Gelfand and Fomin (1963, ch. 8).

9. Recall from Section 3.1 that the stimulus space is always considered an open set, in order to ensure that any point can serve as reference stimulus for a psychometric function. This implies that the interval of $\lambda$ values is open.

10. With the exception of the antecedent property of G5, in Section 3.9, which is, however, of minor importance, for the reasons stated at the end of Section 3.9.

\section{APPENDIX \\ Technical Comments and Proofs}

\section{A1 (Section 2.2)}

This can be shown by Taylor-expanding $\gamma_{x}(x+\Delta x)-\gamma_{x}(x)$ and observing that

$\gamma_{x}(x+\Delta x)-\gamma_{x}(x)=\left.\frac{1}{m !} \frac{d^{m} \gamma_{x}(y)}{d y^{m}}\right|_{y=x} \Delta x^{m}+o\left(\Delta x^{m}\right), \Delta x>0$,

if all derivative of lower orders vanish.

\section{A2 (Section 2.3)}

Indeed, transforming $x \rightarrow \bar{x}$ and putting

$$
\bar{\psi}_{\bar{x}}(\bar{y})=\psi_{x}(y)
$$

we have

$$
\begin{aligned}
\bar{F}(\bar{x}) & =\lim _{\Delta \bar{x} \rightarrow 0+} \frac{\sqrt[4]{\bar{\psi}_{\bar{x}}(\bar{x}+\Delta \bar{x})-\overline{\bar{\psi}_{\bar{x}}(\bar{x})}}}{\Delta \bar{x}} \\
& =\lim _{\Delta x \rightarrow 0+}\left(\frac{\sqrt[\mu]{\psi_{x}(x+\overline{\Delta x})-\psi_{x}(x)}}{\Delta x} \frac{\Delta x}{\Delta \bar{x}}\right)=F(x) \frac{d x}{d \bar{x}},
\end{aligned}
$$

because of which

$$
\bar{G}(\bar{a}, \bar{b})=\int_{\bar{a}}^{\bar{b}} \bar{F}(\bar{x}) d \bar{x}=\int_{\bar{a}}^{\bar{b}} F(x) \frac{d x}{d \bar{x}} d \bar{x}=\int_{a}^{b} F(x) d x=G(a, b) .
$$

\section{A3 (Section 3.4)}

The Euler homogeneity follows from

$$
\begin{aligned}
F(\mathbf{x}, a \mathbf{u}) & =\left.\frac{d \Psi_{\mathbf{x}}(\mathbf{x}+\mathbf{u}(a s))}{d s}\right|_{s=0+} \\
& =\left.a \frac{d \Psi_{\mathbf{x}}(\mathbf{x}+\mathbf{u} s)}{d s}\right|_{s=0+}=a F(\mathbf{x}, \mathbf{u}) .
\end{aligned}
$$

\section{A4 (Section 3.4)}

The neighborhood positivity can be proved as follows. If, for a given point $\mathbf{y}$, one considers all directions $\mathbf{u}$ confined to the Euclidean sphere

$$
|u|^{2}=\sum_{i=1}^{n} u_{i}^{2}=1
$$

then $F(\mathbf{y}, \mathbf{u})>0$ on a closed set of $\mathbf{u}$ values, and

$$
e(\mathbf{y})=\inf _{\mathbf{u}}[F(\mathbf{y}, \mathbf{u})]=\min _{\mathbf{u}}[F(\mathbf{y}, \mathbf{u})]>0 .
$$

It follows that on a closed Euclidean ball $|\mathbf{y}-\mathbf{x}| \leq \rho, \rho>0$,

$$
\varepsilon_{\rho}(\mathbf{x})=\inf _{\mathbf{y}}[e(\mathbf{y})]=\min _{\mathbf{y}}[e(\mathbf{y})]>0
$$

\section{A5 (Section 3.5)}

Property $\mathfrak{I} 1$ follows from the fact that $F(\mathbf{x}(t), \dot{\mathbf{x}}(t))$ is nonnegative, so that $\mathfrak{I}\left[\mathbf{a} x(t)_{\mathbf{b}}\right]=0$ implies $F(\mathbf{x}(t), \dot{\mathbf{x}}(t))=0$ for all $t$, which is only possible if $\dot{x}(t)=0$, which in turn is only possible if $\mathbf{x}(t) \equiv \mathbf{a}=\mathbf{b}$.

Property $\mathfrak{I} 2$ follows from the Euler homogeneity (FF3),

$$
F(\mathbf{x}[\gamma(\tau)], \dot{\gamma}(\tau) \dot{\mathbf{x}}[\gamma(\tau)])=\dot{\gamma}(\tau) F(\mathbf{x}[\gamma(\tau)], \dot{\mathbf{x}}[\gamma(\tau)],
$$

because of which

$$
\begin{aligned}
\left.\mathfrak{S}_{\mathbf{a}} \mathbf{x}[\gamma(\tau)]_{\mathbf{b}}\right] & =\int_{\gamma^{-1}(a)}^{\gamma^{-1}(b)} F(\mathbf{x}[\gamma(\tau)], \dot{\gamma}(\tau) \dot{\mathbf{x}}[\gamma(\tau)]) d \tau \\
& =\int_{a}^{b} F(\mathbf{x}(t), \dot{\mathbf{x}}(t)) d t=\mathfrak{I}\left[_{\mathbf{a}} \mathbf{x}(t)_{\mathbf{b}}\right] .
\end{aligned}
$$

Property $\mathfrak{3} 3$ follows from FF3 and the symmetry property (FF2):

$$
\begin{aligned}
\left.\mathfrak{S}_{\mathrm{b}_{\mathrm{b}}} \mathbf{x}[\gamma(\tau)]_{\mathbf{a}}\right] & =\int_{\gamma^{-1}(b)}^{\gamma^{-1}(a)} F(\mathbf{x}[\gamma(\tau)], \dot{\gamma}(\tau) \dot{\mathbf{x}}[\gamma(\tau)]) d \tau \\
& =\int_{\gamma^{-1}(b)}^{\gamma^{-1}(a)} F(\mathbf{x}[\gamma(\tau)],(-\dot{\gamma}(\tau)(-\dot{\mathbf{x}}[\gamma(\tau)])) d \tau \\
& =\int_{\gamma^{-1}(b)}^{\gamma^{-1}(a)}-\dot{\gamma}(\tau) F(\mathbf{x}[\gamma(\tau)], \dot{\mathbf{x}}[\gamma(\tau)]) d \tau,
\end{aligned}
$$

which transforms into $\mathfrak{I}\left[\mathbf{a} x(t)_{\mathbf{b}}\right]$. 
Property $\mathfrak{9} 4$ follows from the invariance property of the metric function (FF4) on observing that tangent vectors of a curve always transform as contravariant vectors,

$$
\dot{x}^{i}(t)=\sum_{j=1}^{n} \frac{\partial x^{i}(t)}{\partial \bar{x}^{j}(t)} \dot{\bar{x}}^{j}(t), i=1, \ldots, n .
$$

\section{A6 (Section 3.6)}

Property G3 is proved as follows.

Symmetry. Under an arbitrarily chosen negative diffeomorphism $t=\gamma(\tau), \dot{\gamma}(t)<0$, any path $\mathbf{a}(t)_{\mathbf{b}}$ is in a one-to-one correspondence with the path ${ }_{\mathrm{b}} \mathbf{x}(\gamma(\tau))_{\mathrm{a}}$ that has the same value of $\mathfrak{I}$. Therefore, the infima for the two sets, $\left\{_{\mathbf{a}} \mathbf{x}(t)_{\mathbf{b}}\right\}$ and $\left\{_{\mathbf{b}} \mathbf{x}(\gamma(\tau))_{\mathbf{a}}\right\}$, coincide, and since these sets include all possible piecewise continuously differentiable paths from $\mathbf{a}$ to $\mathbf{b}$ and from $\mathbf{b}$ to $\mathbf{a}$, respectively, we have

$$
G(\mathbf{a}, \mathbf{b})=G(\mathbf{b}, \mathbf{a}) .
$$

Triangle inequality. Suppose that for some points $\mathbf{a}, \mathbf{b}, \mathbf{c}$,

$$
\inf _{\mathbf{a}(t)_{\mathbf{b}}}\left\{\mathfrak{I}\left[_{\mathbf{a}} \mathbf{x}(t)_{\mathbf{b}}\right]\right\}>\inf _{\mathbf{a}(t)_{\mathbf{c}}}\left\{\mathfrak{I}\left[_{\mathbf{a}} \mathbf{y}(t)_{\mathbf{c}}\right]\right\}+\inf _{\mathbf{c}(t)_{\mathbf{b}}}\left\{\mathfrak{I}\left[\mathbf{c} \mathbf{z}(t)_{\mathbf{b}}\right]\right\}
$$

Then one should be able to find paths ${ }_{a} \mathbf{x}(t)_{\mathbf{b}},{ }_{\mathbf{a}} \mathbf{y}(t)_{\mathbf{c}}$, and ${ }_{\mathrm{c}} \mathbf{z}(t)_{\mathbf{b}}$ whose $\mathfrak{I}$ values are sufficiently close to the corresponding infima, so that

$$
\mathfrak{I}\left[{ }_{\mathbf{a}} \mathbf{x}(t)_{\mathbf{b}}\right]>\mathfrak{I}\left[_{\mathbf{a}} \mathbf{y}(t)_{\mathbf{c}}\right]+\mathfrak{I}\left[_{\mathbf{c}} \mathbf{z}(t)_{\mathbf{b}}\right]
$$

By three positive diffeomorphic transformations, the domains of the parameter $t$ in the three paths can be made to be $(a, b),(a, c)$, and $(c, b)$, respectively, with $a<c<b$. Then the concatenation of ${ }_{\mathbf{a}} \mathbf{y}(t)_{\mathbf{c}}$, with $\mathbf{z}(t)_{\mathbf{b}}$ forms a path ${ }_{\mathbf{a}} \mathbf{x}^{*}(t)_{\mathbf{b}}$, which is piecewise continuously differentiable and

$$
\mathfrak{I}\left[_{\mathbf{a}} \mathbf{x}^{*}(t)_{\mathbf{b}}\right]<\mathfrak{S}\left[_{\mathbf{a}} \mathbf{x}(t)_{\mathbf{b}}\right]
$$

Since $\mathfrak{I}\left[{ }_{\mathbf{a}} \mathbf{x}(t)_{\mathbf{b}}\right]$ can be chosen arbitrarily close to

$$
\inf _{\mathbf{a}(t)_{\mathbf{b}}}\left\{\mathfrak{I}\left[_{\mathbf{a}} \mathbf{x}(t)_{\mathbf{b}}\right]\right\}
$$

the inequality above implies

$$
\mathfrak{I}\left[\mathbf{a}_{\mathbf{a}} \mathbf{x}^{*}(t)_{\mathbf{b}}\right]<\inf _{\mathbf{a}(t)_{\mathbf{b}}}\left\{\mathfrak{I}\left[_{\mathbf{a}} \mathbf{x}(t)_{\mathbf{b}}\right]\right\}
$$

which is self-contradictory. We conclude that

$$
G(\mathbf{a}, \mathbf{b}) \leq G(\mathbf{a}, \mathbf{c})+G(\mathbf{c}, \mathbf{b}) .
$$

Zero distance property. That

$$
G(\mathbf{a}, \mathbf{b})=0 \text { if } \mathbf{a}=\mathbf{b}
$$

is obvious. To prove the reverse, assume that $\mathbf{a} \neq \mathbf{b}$, but $G(\mathbf{a}, \mathbf{b})=$ 0 . Then there must exist a sequence of curves

$$
\mathbf{a}^{\mathbf{x}^{(k)}(l)_{\mathbf{b}}, k=1,2, \ldots,}
$$

such that

$$
\int_{0}^{L^{(k)}} F\left(\mathbf{x}^{(k)}(l), \dot{\mathbf{x}}^{(k)}(l)\right) d l \rightarrow 0 \text { as } k \rightarrow \infty,
$$

where $l$ is chosen to represent the Euclidean length of the arc measured from point a, $L^{(k)}$ being the Euclidean length of the entire path. With this choice of the curve parameter,

$$
\left|\dot{\mathbf{x}}^{(k)}(l)\right|^{2}=\sum_{i=1}^{n}\left(\dot{x}_{i}^{(k)}(l)\right)^{2}=1,
$$

which follows from the obvious identity

$$
\sum_{i=1}^{n}\left(\mathrm{~d} x_{i}^{(k)}(l)\right)^{2}=\mathrm{d} l^{2} .
$$

One can therefore invoke Property FF5 of Section 3.4 to state that

$$
\varepsilon_{\rho}(\mathbf{a})>0
$$

for a closed ball centered at $\mathbf{a}$ with a radius

$$
\rho<|\mathbf{b}-\mathbf{a}| \text {. }
$$

Any path $\mathbf{a}^{(k)}(l)_{\mathbf{b}}$ must puncture the sphere of the ball. Denoting the Euclidean length of the $\operatorname{arc~of} \mathbf{x} \mathbf{x}^{(k)}(l)_{\mathbf{b}}$ contained within the ball by $l^{(k)}$, we must have

$$
\int_{0}^{l^{(k)}} F\left(\mathbf{x}^{(k)}(l), \dot{\mathbf{x}}^{(k)}(l)\right) d l \rightarrow 0 \text { as } k \rightarrow \infty .
$$

This is, however, impossible, because

$$
\int_{0}^{l^{(k)}} F\left(\mathbf{x}^{(k)}(l), \dot{\mathbf{x}}^{(k)}(l)\right) d l \geq \varepsilon_{\rho}(\mathbf{a}) l^{(k)} \geq \varepsilon_{\rho}(\mathbf{a}) \rho .
$$

The proof of G3 is complete.

\section{A7 (Section 3.8)}

For any positive $a$, the identity

$$
F(\mathbf{x}, a \mathbf{u})=f[\bar{F}(\mathbf{x}, a \mathbf{u})]
$$

implies

$$
a F(\mathbf{x}, \mathbf{u})=f[a \bar{F}(\mathbf{x}, \mathbf{u})],
$$

which can also be written as

$$
a f[(\bar{F}(\mathbf{x}, \mathbf{u})]=f[a \bar{F}(\mathbf{x}, \mathbf{u})] .
$$

Putting $\bar{F}(\mathbf{x}, \mathbf{u})=1$, we get

$$
a f(1)=f(a),
$$

and the proof obtains by denoting $f(1)=k$ and observing that it must be positive.

(Manuscript received December 17, 1997; revision accepted for publication June 2, 1998.) 